\title{
Microtubules stabilize intercellular contractile force transmission during tissue folding
}

\author{
Clint S. Ko ${ }^{1}$, Vardges Tserunyan ${ }^{1}$, and Adam C. Martin ${ }^{1}$ \\ ${ }^{1}$ Department of Biology, \\ Massachusetts Institute of Technology, Cambridge, MA 02142, USA
}

Corresponding author \& Lead Contact:

Adam C. Martin

acmartin@mit.edu

31 Ames St.

Cambridge, MA 02142, USA 


\section{Abstract}

During development, forces transmitted between cells are critical for sculpting epithelial tissues. Actomyosin contractility in the middle of the cell apex (medioapical) can change cell shape (e.g., apical constriction), but can also result in force transmission between cells via attachments to adherens junctions. How actomyosin networks maintain attachments to adherens junctions under tension is poorly understood. Here, we discovered that microtubules stabilize actomyosin intercellular attachments in epithelia during Drosophila mesoderm invagination. First, we used live imaging to show a novel arrangement of the microtubule cytoskeleton during apical constriction: medioapical, non-centrosomal Patronin (CAMSAP) foci formed by actomyosin contraction organizes an apical microtubule network. Microtubules were required for mesoderm invagination but were not necessary for apical contractility or adherens junction assembly. Instead, microtubules promoted the stable connection between medioapical actomyosin and adherens junctions. These results define a role for coordination between actin and microtubule cytoskeletal systems in intercellular force transmission and tissue morphogenesis. 


\section{Introduction}

Apical constriction is a ubiquitous cell shape change that results in dramatic rearrangements of tissue architecture, such as tissue folding (Sawyer et al., 2010; Heisenberg and Bellaïche, 2014; Martin and Goldstein, 2014). The cellular force necessary to constrict a cell apex is generated by actomyosin contraction, which is regulated by RhoA signaling (Kasza and Zallen, 2011; Lecuit et al., 2011). During apical constriction, the apical cortex is often polarized; myosin-II (myosin) is activated near the middle of the apical cortex (medioapical), which contracts an actin filament (F-actin) network that spans the apical surface (Sawyer et al., 2009; Blanchard et al., 2010; David et al., 2010; Mason et al., 2013; Booth et al., 2014; Sánchez-Corrales et al., 2018). In order for these changes in cell geometry to cause tissue morphogenesis, cellular forces must be transmitted and integrated across the tissue (FernandezGonzalez et al., 2009; Lecuit and Yap, 2015). This is mediated by connecting contractile actomyosin meshworks to E-cadherin-based adherens junctions (Martin et al., 2010, Sawyer et al., 2011). Molecular components that mediate this linkage have been identified and are important for morphogenesis (Sawyer et al., 2009; Desal et al., 2013). In addition, this attachment has been shown to be dynamic and actin turnover is required to stabilize attachments by repairing lost connections (Roh-Johnson et al., 2012; Jodoin et al., 2015). However, whether other mechanisms maintain actomyosin network connections to junctions, in the face of tension, remains unknown.

During gastrulation in the early Drosophila embryo, apical constriction leads to mesoderm folding and cell invagination (Leptin and Grunewald, 1990; Sweeton et al., 1991) (Fig. 1 A). Mesoderm cells express transcription factors, Twist and Snail, that 
promote apical RhoA GTPase activation, which induces actomyosin contractility (Barrett and Settleman, 1997; Häcker and Perrimon, 1998; Dawes-Hoang et al., 2005; Fox and Peifer, 2007; Kölsch et al., 2007). Contractile force is transmitted across the folding tissue through adherens junctions, resulting in epithelial tension predominantly along the anterior-posterior axis (Martin et al., 2010; Chanet et al., 2017). Apical constriction in multiple invagination processes depends on polarized RhoA signaling, with active RhoA and its downstream effector Rho-associated coiled-coil kinase (ROCK) that activates myosin (Amano et al., 1996; Mizuno et al., 1999) being enriched in the middle of the apical surface (Mason et al., 2013; Booth et al., 2014; Coravos et al., 2016; Chung et al., 2017). It is poorly understood how intercellular actomyosin connections are stabilized when the medioapical pool of active RhoA is present at a distance from cell junctions.

While the regulation of actomyosin contractility during apical constriction has been well-studied, the organization and importance of the microtubule cytoskeleton is less well understood. In epithelia, there is evidence for different microtubule functions, such as regulating assembly or position of the adherens junctions (Harris and Peifer, 2005; Stehbens et al., 2006; Meng et al., 2008; Le Droguen et al., 2015), recruiting apical myosin (Booth et al., 2014), regulating RhoA (Rogers et al., 2004; Nagae et al., 2013), and providing a counter-force resisting actomyosin contraction (Takeda et al., 2018; Singh et al., 2018). Importantly, studies have shown that microtubules are necessary for cell shape changes, like apical constriction (Lee and Harland, 2007; Lee et al., 2007; Booth et al., 2014). However, many of these studies involved depolymerizing or eliminating the entire microtubule cytoskeleton, making it difficult to 
determine if microtubules play other roles. Given the critical role of actin and myosin dynamics during morphogenesis, it remains to be seen whether microtubule organization might play additional roles in apical constriction and/or intercellular connectivity.

Here, we investigated microtubule cytoskeleton organization and its mechanistic contribution to mesoderm invagination. We show that a microtubule minus-end-binding protein, Patronin, co-localizes with myosin in the medioapical cortex of apically constricting cells. We found that microtubules are required for mesoderm invagination, but not apical cortex contractility. Instead, proper microtubule organization stabilizes intercellular contractile force transmission. Finally, we determined actomyosin contractility drives the organization of the microtubule cytoskeleton, suggesting feedback between actomyosin and microtubules that maintains apical actin cortex attachment to the adherens junctions. 


\section{Results}

\section{Medioapical Patronin focus co-localizes with myosin in apically constricting cells}

To determine if the microtubule cytoskeleton plays a role in the apical constriction of mesoderm cells of the early Drosophila embryo, we first sought to establish the organization of the microtubule cytoskeleton. In most polarized epithelial cells, linear arrays of microtubules align with the apical-basal axis such that the minus ends are uniformly localized across the apical surface (Bacallao et al., 1989; Khanal et al., 2016; Toya et al., 2016). These minus ends are often capped and stabilized by a family of calmodulin-regulated spectrin-associated proteins (CAMSAPs or Patronin in Drosophila), which specifically bind to microtubule minus ends (Goodwin and Vale, 2010; Khanal et al., 2016; Noordstra et al., 2016; Toya et al., 2016). We imaged live and fixed Drosophila embryos expressing Patronin::GFP driven by the ubiquitin promoter (Ubi-p63E-Patronin::GFP, herein referred to as Patronin::GFP). During gastrulation, there was a striking difference in Patronin::GFP localization between mesoderm (apically constricting) and ectoderm cells (not constricting) (Fig. 1 B). Specifically, Patronin::GFP was polarized to a central focus in the medioapical cell cortex of mesoderm cells, but was enriched at cell junctions in the ectoderm (Fig. 1, B and C; Movie 1).

To demonstrate the difference in Patronin localization between these cell types, we measured the ratio of average junctional Patronin::GFP intensity to the average medial Patronin::GFP intensity. During mesoderm invagination, there was a significant difference in the ratio of junctional to medial Patronin::GFP intensity between mesoderm 
and ectoderm cells, with medial Patronin::GFP intensity being highest in the mesoderm (mean junctional to medial ratio of $0.75 \pm .53$ compared to $4.75 \pm 3.47$; Fig. 1 D). In addition, the maximum medioapical Patronin::GFP intensity was higher in the mesoderm than the ectoderm, consistent with Patronin forming a large apical focus in these cells (Fig. 1 E). Patronin::GFP in the ectoderm formed smaller puncta that were distributed across the apical cortex (Fig. 1 B). Earlier in development, from syncytial to early cellularization stages, Patronin::GFP localized to the two centrosomes above the nucleus (Fig. 1 C). During mid-late cellularization, Patronin::GFP shifted to a more apical localization at cell junctions in both the mesoderm and ectoderm (Fig. $1 \mathrm{C}$ and Movie 1), consistent with other studies of this developmental stage (Takeda et al., 2018).

Medioapical Patronin::GFP foci localized to the center of medioapical myosin patches (Fig. $1 \mathrm{~F})$. This localization mirrors that of active RhoA and ROCK (Mason et al., 2013; Mason et al., 2016). Interestingly, apical constriction of the posterior midgut cells (i.e. the endoderm) also exhibited medioapical Patronin::GFP enrichment (Fig. 1 G). These results demonstrated that medioapical Patronin localization is a fundamental organization shared by apically constricting cells in the early Drosophila embryo.

\section{Medioapical Patronin stabilizes non-centrosomal microtubules}

Given the striking and centralized Patronin::GFP localization in mesoderm cells, we next determined if this structure represented a centrosome or another type of microtubule organizing center. First, we fixed embryos and detected $\alpha$-Tubulin by immunofluorescence. When viewing cells en face, mesoderm cells displayed both 
punctate and fiber-like structures, suggesting that microtubule bundles are arranged parallel and perpendicular to the apical surface (Fig. 2 A and B). In contrast, the ectoderm had significantly fewer medioapical microtubules at the time of mesoderm invagination (Fig. 2 B). Because fixation often destroys dynamic medioapical cytoskeletal networks, we next visualized microtubules in live embryos expressing GFPtagged CLIP170, which gave us the best labeling of microtubules at this stage. During early tissue folding, we observed dense patches of GFP::CLIP170 puncta that colocalized with apical myosin (Fig. 2 C and Movie 2). Given the co-localization between myosin and Patronin, these CLIP170 patches are likely co-localized with Patronin and represent an apical microtubule-organizing center.

Apical Patronin is often associated with non-centrosomal microtubules in epithelia (Noordstra et al., 2016; Toya et al., 2016). To test whether these Patronin foci were centrosomes, we examined the position of centrosomal markers, which were significantly below the apical surface throughout folding (Fig. S1, A and B). We observed lower levels of Patronin::GFP associated with centrosomes, which were wellseparated from medioapical Patronin::GFP foci (Fig. S1 B). This localization could reflect sites of active Patronin-mediated minus-end stabilization of microtubules that are released from centrosomes, similar to a proposed function of ninein (Mogensen et al., 2000; Moss et al., 2007), or a separate centrosomal pool of Patronin. Thus, there is a distinct organization of non-centrosomal microtubules at the apical cortex during mesoderm invagination.

A well-known function of CAMSAPs is to stabilize microtubules (Goodwin and Vale, 2010; Tanaka et al., 2012; Hendershott and Vale, 2014; Jiang et al., 2014). To 
determine whether Patronin stabilizes non-centrosomal microtubules near the apical cortex, we fixed Patronin-depleted embryos and immunostained with antibodies against acetylated- $\alpha$-Tubulin, a marker of stable microtubule polymers (Westermann and Weber, 2003). We verified that RNAi depleted Patronin protein levels by Western blot (Fig. S1 C). Control or wild-type embryos exhibited acetylated microtubules that are both lying across the apical surface and parallel to the apical-basal axis (Fig. 2 D). In contrast to control knock-down, Patronin depletion dramatically reduced visible bundles of apical acetylated-Tubulin in mesoderm cells (Fig. 2 D). This result suggested that Patronin stabilizes apical, non-centrosomal microtubules.

To determine whether Patronin localization required microtubules, we injected Patronin::GFP expressing embryos with the microtubule depolymerizing drug, colchicine. Colchicine injection dramatically disrupted Patronin localization (Fig. 2 E), consistent with the reported loss of polarized CAMSAP3 localization in Caco-2 cells treated with nocodazole, another inhibitor of microtubule polymerization (Toya et al., 2016). Together, these results suggested that medioapical Patronin foci form a noncentrosomal microtubule-organizing center in constricting mesoderm cells and that Patronin and microtubule localization is interdependent.

\section{Patronin puncta coalesce into foci during myosin pulses}

During mesoderm invagination, apical myosin initially accumulates in cycles of assembly and disassembly and these pulses are associated with apical constriction (Martin et al., 2009; Vasquez et al., 2014). To determine how medioapical Patronin foci form, we analyzed the spatiotemporal dynamics of Patronin::GFP relative to myosin. In 
contrast to myosin, we did not observe clear cycles of assembly and disassembly for Patronin::GFP puncta (Fig. 3 A and Movie 3). Instead, medioapical Patronin foci appeared to grow by the continuous coalescence of smaller Patronin::GFP puncta (Fig. 3 A). To determine if Patronin::GFP coalescence was associated with apical myosin contraction, we identified individual myosin pulses and looked at the behavior of Patronin::GFP in the region of the pulse. During myosin pulse assembly, we usually observed instances of Patronin::GFP coalescence, which formed a brighter, more compact focus (Fig. 3 B). When we analyzed the maximum intensity profiles of myosin and Patronin averaged across 20 pulses, we found that myosin intensity peaked about 5 seconds before Patronin::GFP intensity (Fig. 3 C). These data suggested that apical contractility drives formation of medioapical Patronin foci, which we showed co-localize with apical myosin patches (Fig. $1 \mathrm{~F})$.

During myosin pulse disassembly, there was a local decrease in Patronin::GFP intensity as the more compact Patronin focus sometimes appeared to relax and revert back to smaller puncta (Fig. 3, C and D). To determine how closely changes in Patronin intensity track with myosin intensity, we analyzed the time-resolved cross-correlation between changes in Patronin::GFP and myosin intensities. We identified a significant correlation occurred between these two signals, with the maximum correlation occurring at a time offset of -3.9 seconds, consistent with the myosin signal preceding Patronin signal (Fig. 3 E). These results demonstrated a tight correlation between myosin and Patronin intensity with myosin preceding Patronin coalescence, suggesting that actomyosin contraction could form medioapical Patronin foci in mesoderm cells. 


\section{Actomyosin contraction forms a medioapical, non-centrosomal microtubule- organizing center}

Because microtubule/Patronin organization in the mesoderm resembled that of active RhoA and was correlated with myosin contractility (Fig. 3) (Mason et al. 2013; Mason et al., 2016), we tested whether RhoA and myosin contractility were required for apical microtubule cytoskeleton organization. To inhibit myosin activation, we injected embryos with the ROCK inhibitor, Y-27632 (Royou et al; 2002; Dawes-Hoang et al., 2005). In comparison to water-injected embryos, Y-27632 disrupted Patronin::GFP apical localization (Fig. 4, A and B). However, because Y-27632 also inhibits aPKC (Davies et al., 2000), we additionally analyzed Patronin localization after inhibiting RhoA activity with the C3-exoenzyme (Crawford et al., 1998). RhoA inhibition similarly disrupted apical Patronin (Fig. 4, C and D), suggesting that RhoA activity and myosin contractility promotes the formation of a medioapical microtubule-organizing center.

Because RhoA also promotes contraction via F-actin assembly (Großhans et al., 2005; Fox and Peifer, 2007; Murrell et al., 2015; Agarwal and Zaidel-Bar, 2018), we tested whether disrupting the apical F-actin network affects microtubule organization. We tested F-actin's requirement by injecting drugs that interfere with F-actin assembly, Cytochalasin D (CytoD) and Latrunculin B (LatB). Similar to Y-27632 and C3exoenzyme injections, CytoD and LatB both disrupted medioapical Patronin focus formation. In these embryos, Patronin puncta did not coalesce, instead remaining as small, dynamic puncta (Fig. 4 E; Fig. S2 B). Importantly, it has been shown that in 
Caco-2 cells, where CAMSAP-3 localizes as puncta in the apical cortex, F-actin depolymerization reduces CAMSAP-3 puncta in cortical regions (Toya et al., 2016).

Notably, F-actin depolymerization resulted in a different organization of the microtubule cytoskeleton as it formed prominent bundles that lay parallel to the apical surface (Fig.

S2 A) Altogether, our data indicated that RhoA activity, which leads to actomyosin contraction, is required to organize apical, non-centrosomal microtubules during mesoderm invagination.

\section{Microtubules are not required for apical contractility or adherens junction}

\section{assembly}

Studies in other developmental contexts have suggested that microtubules are necessary for cell invagination (Lee et al., 2007; Booth et al., 2014). We found that microtubules were also important for mesoderm cell invagination (Fig. 5 A; Movie 4). One way in which microtubules could promote mesoderm cell invagination is to induce contractility, such as by activating apical actomyosin assembly (Rogers et al., 2004; Booth et al., 2014). Alternatively, microtubules could regulate adherens junction assembly or position (Harris and Peifer, 2005; Stehbens et al., 2006; Meng et al., 2008; Le Droguen et al., 2015). A final possibility is that microtubules regulate the connection between actomyosin networks and the adherens junctions.

To determine whether microtubules are required to induce contractility in mesoderm cells, we disrupted microtubules pharmacologically or by gene depletion. Despite tissue folding failure, we found that colchicine injection did not disrupt apical myosin activation or the initiation of apical constriction (19/19 embryos imaged) (Fig. 5 
A; Movie 4). To demonstrate that apical constriction occurred, we measured apical area at the onset of constriction and $\sim 5$ minutes later for control and drug-injected embryos (Fig. 5 B). Depleting Patronin by RNAi also disrupted tissue folding (11/44 embryos imaged) without affecting apical myosin localization or constriction onset (44/44 embryos imaged) (Fig. S3 A).

To specifically test whether microtubule dynamics/organization were important for apical force generation, we injected embryos with taxol, a microtubule-stabilizing agent. Taxol injection disrupted apical microtubule organization, resulting in thick bundles that crossed the apical surface (Fig. S3 D). Perturbing microtubule dynamics/organization with taxol disrupted folding, but again, had no effect on initial cell constrictions or myosin accumulation (21/21 embryos imaged) (Fig. 5, B and C). Furthermore, taxol-injected embryos exhibited a clear cross-correlation peak between apical constriction rate and the rate of change in myosin intensity, which is indicative of myosin pulsing (Fig. 5 D) (Mason et al., 2013; Vasquez et al., 2014). Finally, the polarized localization of ROCK was unaffected in both colchicine- and taxol-injected embryos (Fig. $5 \mathrm{E}$ ). These data suggested that microtubule organization is not important for actomyosin activation or the onset of apical constriction.

To determine if microtubules were required for apical adherens junction assembly (Dawes-Hoang et al., 2005; Stehbens et al., 2006; Kölsch et al., 2007; Marston et al., 2016; Weng and Wieschaus, 2016), we analyzed E-cadherin in live embryos injected with microtubule drugs or depleted of Patronin. There were no defects in apical E-cadherin polarity in either case (Fig. 5 F; Fig. S3, B and C). Thus, during mesoderm invagination, microtubules are not necessary to induce contractility (i.e., 
apical myosin activation) or to assemble apical adherens junctions, but are required for tissue folding.

\section{Proper microtubule organization stabilizes intercellular force transmission}

In the previous section, we showed that apical myosin and adherens junction accumulation was unaffected in Patronin-depleted, colchicine-, and taxol-injected embryos. A remaining possibility was that microtubules mediate the connection between actomyosin and adherens junctions (Roh-Johnson et al., 2012; Jodoin et al., 2015).

Therefore, we analyzed actomyosin attachments to adherens junctions and the resulting intercellular connectivity between actomyosin networks after microtubule cytoskeleton disruption. In both colchicine- and taxol-injected embryos, the apical myosin networks exhibited striking separations (Fig. 6, A and B; Fig. S4 A; Movie 5). Myosin spots in different cells chaotically separated and then moved back together, similar to perturbations that affect the stability of actomyosin network connections to adherens junctions (Fig. 6 B and Movie 5) (Mason et al., 2013; Jodoin et al., 2015). These separations only occurred after a significant buildup of apical myosin and the formation of a supracellular myosin network, suggesting that microtubules are important for a later step in the folding process when there is significant epithelial tension.

The loss of intercellular connectivity of actomyosin after microtubule organization disruption was not associated with a disorganized apical cortex. When we injected embryos expressing GFP-tagged ROCK with taxol, we saw that medial enrichment of ROCK was unaffected even when there were clear separations (Fig. 6 C). We also analyzed junctional E-cadherin localization after microtubule disruption. We previously showed that E-cadherin exhibited proper junctional localization after colchicine injection 
or Patronin depletion (Fig. 5 F; Fig. S3, B and C). After taxol injection, we found that Ecadherin persists at the cell-cell interface during myosin separations (Fig. S4 A). Moreover, actomyosin network separations could be repaired as myosin in separate cells re-established connections and pulled back together (Fig. 6 B; Fig. S4 A; Movie 5), which further suggested the presence of functional adherens junctions. In contrast, Ecadherin depletion or depleting proteins that link actin to adherens junctions resulted in a complete separation of actomyosin networks from cell junctions without repair (Sawyer et al., 2009; Martin et al., 2010). Only at much later stages, when the integrity of the tissue had declined, did we observe E-cadherin mis-localized across the apical cortex (Fig. S4 B).

To determine that the observed drug-injection phenotypes were not caused by disrupting a previous developmental process, such as cellularization, we injected drugs during tissue folding. For most experiments resulting in apical myosin separations, microtubule inhibitors were injected at middle to late cellularization. However, injecting colchicine into live embryos that had completed cellularization and started apical constriction (i.e., injected when apical myosin present) resulted in the same phenotype (2/2 embryos imaged) (Fig. 6 D). Thus, microtubules are required during folding to maintain actomyosin attachments to adherens junctions.

Finally, to more specifically disrupt apical microtubule organization, we perturbed microtubules by depleting Patronin by RNAi, which we showed destabilized apical noncentrosomal microtubules (Fig. 3 D). In 11 out of 44 knockdowns, we also observed dynamic tearing of the myosin network (Fig. 6 E). Overall, our results demonstrated that microtubules are not required for medioapical myosin activation or adherens junction 
bioRxiv preprint doi: https://doi.org/10.1101/540344; this version posted February 4, 2019. The copyright holder for this preprint (which was not certified by peer review) is the author/funder, who has granted bioRxiv a license to display the preprint in perpetuity. It is made available under aCC-BY-NC-ND 4.0 International license.

assembly, but are critical for actomyosin networks in adjacent cells to stably transmit

force to each other across adherens junctions (Fig. 6 F). 


\section{Discussion}

Our work identifies a role for the microtubule cytoskeleton in promoting the stable transmission of force between epithelial cells during mesoderm invagination in Drosophila embryos. We have demonstrated, to our knowledge, a novel medioapical organization to the microtubule cytoskeleton, where a medioapical focus of the microtubule minus-end-binding protein Patronin is present in apically constricting cells. Patronin puncta coalesce to form medioapical Patronin foci, which depends on actomyosin contraction. Patronin stabilizes apical, non-centrosomal microtubules, but these are dispensable for apical myosin polarity, apical E-cadherin, and initiation of apical constriction. In contrast, microtubules were required to stabilize medioapical actomyosin network attachment to E-cadherin-based cell junctions. Disrupting microtubules resulted in dynamic separations between myosin and intercellular junctions that prevented mesoderm invagination. This study uncovers a previously unrecognized role for microtubule organization in integrating contractile forces across a tissue during morphogenesis.

\section{Apical constriction in the early Drosophila embryo is associated with a}

\section{medioapical, non-centrosomal microtubule-organizing center}

Apically constricting cells in the mesoderm and endoderm shared a similarly polarized microtubule organization with Patronin foci localized to the medioapical cortex. In addition, live imaging of embryos expressing GFP::CLIP170 revealed the appearance of dense patches of microtubules in the mesoderm that co-localized with apical myosin patches. Given the high concentration of microtubule-associated proteins that localize to 
the medioapical focus, this appears to be a non-centrosomal microtubule-organizing center. It is unclear whether microtubules radiate out from this organizing center with growing plus ends enriched towards cell edges, such as the organization that has been observed for centrosomal microtubules in MCF-7, MDCK, and hE-CHO cells (Stehbens et al., 2006). Both fixed and live data suggested that microtubules span the apical cortex and we sometimes observed CLIP170 puncta at cell junctions. In addition, after taxol injection, microtubules formed thick bundles that appeared to extend across cell boundaries, similar to the supracellular myosin fibers that form during mesoderm invagination (Martin et al., 2010). Together, these results suggested that the medioapical cortex is a zone of stable microtubules that could act as an organizing center from which a population of microtubules grow towards cell junctions (Fig. 7 A).

Patronin foci co-localized with apical myosin patches and formed by coalescence that spatiotemporally correlated with myosin pulses. During myosin pulses, peak Patronin intensity was observed $\sim 4$ seconds after peak myosin intensity. Thus, polarized Patronin organization may be a consequence of medioapical actomyosin contraction. In support of this, injecting higher doses of CytoD/LatB, which leads to the formation of small myosin and ROCK puncta that fail to coalesce (Mason et al., 2013; Coravos et al., 2016), causes Patronin to remain as small puncta that do not coalesce (Fig. 7, A and B). One possible connection between Patronin and actin is the spectrin cytoskeleton because spectrin associates with F-actin and Patronin has been shown to associate with spectrin isoforms (King et al., 2014; Khanal et al., 2016). However, Patronin's central localization also depends on microtubules and it is possible that microtubules trapped in the actin network are 'collected' by actomyosin contractile flow 
(Salmon et al., 2002). To our knowledge, this is the first example of actomyosin contraction forming a microtubule-organizing center.

\section{Microtubules are not required for apical myosin activation in mesoderm cells}

Apical constriction and subsequent tissue invagination have been shown to be dependent on microtubules in bottle cells during Xenopus laevis gastrulation and placodal cells during salivary gland tubulogenesis in Drosophila (Lee and Harland, 2007; Booth et al., 2014). In the salivary gland cells, microtubules were required for the formation of a medioapical actomyosin network that constricted the cell apex (Booth et al., 2014), consistent with a proposed model for microtubule-dependent activation of RhoA at the apical cortex (Rogers et al., 2004). Apical cortex organization leading to polarized domains of active contractility is important during apical constriction (Mason et al., 2013; Booth et al., 2014; Coravos et al., 2016). However, in mesoderm cells, depolymerizing microtubules or disrupting microtubule dynamics/organization did not lead to a loss of apical myosin activation and initiation of apical constriction was normal. Moreover, medioapical ROCK polarity was unaffected after taxol injection. Overall, these data suggest that during mesoderm invagination in the early embryo, medioapical RhoA activity is not affected by microtubule perturbation. However, RhoA activity was important for microtubule organization (Fig. 7 A).

\section{Microtubules stabilize actomyosin attachment to adherens junctions}

We found that disrupting microtubules leads to a myosin separation phenotype that is similar to that observed in embryos with disrupted actin turnover (Jodoin et al., 2015), 
indicative of a loss of stable actomyosin network attachment to adherens junctions.

When F-actin is disrupted with low doses of the depolymerizing drugs CytoD or LatB, the medioapical signaling center of ROCK/myosin separates from the junctions (Mason et al., 2013; Jodoin et al., 2015). Since disruption of either F-actin or microtubules leads to a similar phenotype and F-actin depolymerization affects the polarized localization of ROCK and Patronin, this suggests a complex crosstalk between different cytoskeletal networks that act together to organize the apical cortex and promote stable force transmission between cells.

Microtubules may stabilize actomyosin attachments to adherens junctions by physical association with the apical F-actin network. Such an association could be mediated by actin-microtubule crosslinkers (Applewhite et al., 2010; Girdler et al., 2016; Takács et al., 2017). When we injected embryos with CytoD or LatB, Patronin localized as small, dynamic puncta across the apical surface of cells, similar to myosin (Mason et al., 2013). Many of these Patronin and myosin puncta co-localized (Fig. 7 B), suggesting an association between actomyosin and microtubules. Additionally, apical microtubules may aid or promote actin polymerization or actin bundling (López et al., 2014; Henty-Ridilla et al., 2016). These interactions may play a role in repairing F-actin meshwork holes to promote reattachment (Jodoin et al., 2015). While the importance of microtubules for apical constriction initiation could be tissue-specific, it will be important to investigate if microtubules might stabilize actomyosin connections to intercellular junctions in other developmental contexts. 


\section{Materials and Methods}

\section{Fly stocks and genetics}

Fly stocks and crosses used in this study are listed in Table S1. Control (Rhodopsin 3) and Patronin knockdown flies were generated by crossing virgins of the shRNA lines to male flies carrying maternally-loaded Gal4 drivers with appropriate markers. Crosses were maintained at $27^{\circ} \mathrm{C}$. In the F2 generation, non-balancer females and males were used to set up cages that were incubated at $25^{\circ} \mathrm{C}$. All other crosses and cages were maintained at $25^{\circ} \mathrm{C}$.

\section{Live and fixed imaging}

For live imaging, embryos were dechorionated in $50 \%$ bleach, washed in water, and mounted onto a glass slide coated with glue (double-sided tape dissolved in heptane). Coverslips (No. 1.5) coated in glue were attached to the slide to use as spacers and a No. 1 coverslip was attached on top to create a chamber. Halocarbon 27 oil was used to fill the chamber. All imaging took place at room temperature $\left(\sim 23^{\circ} \mathrm{C}\right)$.

For fixed imaging, embryos were dechorionated in bleach, washed in water, then fixed in $8 \%$ paraformaldehyde in $0.1 \mathrm{M}$ phosphate buffer at $\mathrm{pH} 7.4$ with $50 \%$ heptane for 30 minutes and manually devitellinized. For the best microtubule staining, embryos fixed in PFA were devitellinized by removing fixative, adding $50 \%$ methanol, and vortexing. These embryos were stored in $100 \%$ methanol at $-20{ }^{\circ} \mathrm{C}$ and rehydrated in $.01 \%$ Tween 20 in PBS (PBS-T). In addition, some embryos (Fig. S3 B) were heat-fixed (HF) in boiled Triton salt solution (.03\% Triton $\mathrm{X}-100$ and $0.4 \% \mathrm{NaCl}$ in water), cooled on ice, and devitellinized in a 1:1 heptane/methanol solution and stored and rehydrated as above.

Embryos were washed in PBS-T, blocked with 10\% BSA in PBS-T, and incubated with antibodies diluted in PBS-T. To visualize F-actin, manually devitellinized embryos were incubated with Alexa Fluor 647 conjugated phalloidin (Invitrogen) diluted in $5 \%$ BSA in PBS-T overnight at $4{ }^{\circ} \mathrm{C}$. For PFA-fixed and manually devitellinized embryos, Asterless was recognized using antibody (a gift from J. Raff) diluted at 1:500 and $\gamma$-Tubulin (Sigma-Aldrich, GTU-88) at 1:500. For PFA-fixed embryos devitellinized by methanol addition and vortexing, $\alpha$-Tubulin was recognized using antibody (SigmaAldrich) diluted at 1:500 and acetylated-tubulin antibody (Sigma-Aldrich, 6-11B-1) at 1:500. For HF embryos, Armadillo was recognized using antibody (Developmental Studies Hybridoma Bank) diluted 1:500 and Snail using antibody (a gift from M. Biggin) at 1:500. Embryos were incubated with primary antibodies at room temperature for 2 hours. Secondary antibodies used were Alexa Fluor 488, 568, or 647 (Invitrogen) diluted at 1:500 in 5\% BSA in PBS-T incubated overnight at $4{ }^{\circ} \mathrm{C}$. Endogenous GFP signal was visualized for Patronin::GFP. After antibody incubation, embryos were mounted onto glass slides using AquaPolymount (Polysciences, Inc.).

All images were taken on a Zeiss LSM 710 confocal microscope with a 40x/1.2 Apochromat water objective lens, an argon ion, $561 \mathrm{~nm}$ diode, $594 \mathrm{~nm} \mathrm{HeNe}, 633 \mathrm{HeNe}$ lasers, and Zen software. Pinhole settings ranged from $1-2.5$ airy units. For two-color live imaging, band-pass filters were set at $\sim 490-565 \mathrm{~nm}$ for GFP and $\sim 590-690 \mathrm{~nm}$ for $\mathrm{mCH}$. For three-color imaging, band-pass filters were set at $\sim 480-560 \mathrm{~nm}$ for GFP, $\sim 580-635 \mathrm{~nm}$ for Alexa Fluor 568, and $\sim 660-750 \mathrm{~nm}$ for Alexa Fluor 647. 


\section{Image processing and analysis}

All images were processed using MATLAB (MathWorks) and FIJI

(http://fiji.sc/wiki/index.php/Fiji). A Gaussian smoothing filter (kernel = 1 pixel) was applied. Apical projections are maximum intensity Z-projections of multiple $z$ sections (2-4 $\mu \mathrm{m})$ and sub-apical sections are optical slices that are $1-2 \mu \mathrm{m}$ below the apical sections.

Image segmentation for quantifications of cell area and myosin and Patronin intensities was performed using custom MATLAB software titled EDGE (Embryo Development Geometry Explorer; https://github.com/mgelbart/embryo-developmentgeometry-explorer; Gelbart et al., 2012). To calculate the junctional to medial ratio of Patronin::GFP intensity (Fig. 1 D), raw images were processed via background subtraction to remove cytoplasmic Patronin::GFP by subtracting the mean plus one half standard deviation intensity value for the first time step from every pixel in all images. We made maximum intensity projections via FIJI and imported the image stacks into EDGE. Cell boundaries were automatically detected and manually corrected, after which EDGE exported cell area and integrated intensity of Patronin::GFP for each cell. Medial Patronin::GFP intensity ( $\left.I_{M, I n t}\right)$ was defined as the integrated intensity value for the whole cell $\left(I_{W, \text { Int }}\right)$ excluding the outermost 2 layers of pixels. Junctional Patronin::GFP intensity $\left(I_{J, \text { Int }}\right)$ was calculated as the difference between $I_{W, \text { Int }}$ and $I_{M, \text { Int }}$. EDGE exported area values as total pixel numbers comprising the region of interest for both the whole cell $\left(S_{W}\right)$ and the medial region $\left(S_{M}\right)$. The area of the junctional region $\left(S_{J}\right)$ was defined as the difference between $S_{W}$ and $S_{M}$. Then we proceeded to calculate the average pixel intensity of the junctional region ( $\left.i_{J}\right)$ to that of the medial region ( $\mathrm{i}_{M}$ ) as follows:

$$
r=\frac{i_{J}}{i_{M}}=\frac{I_{J, I n t}}{I_{M, I n t}} * \frac{S_{M}}{S_{J}}
$$

We used average per pixel intensity values instead of the integrated intensity values for that region to more accurately represent the local protein concentration.

To quantify changes in cell area and myosin intensity (Fig. 5, C and D), raw images were processed via background subtraction as described above. We made maximum intensity projections via FIJI and imported the image stacks into EDGE. Cell boundaries were automatically detected and manually corrected, after which EDGE exported cell area and integrated intensity of Myosin::GFP for each cell. To calculate the cross-correlation between the rate of change in cell area constriction and per pixel myosin intensity buildup rate (Fig. $5 \mathrm{E}$ ), we first smoothed the area and myosin integrated intensity curves of each cell by a moving average ( 3 time steps wide) and then used these values to calculate the average per pixel intensity through time. Then, we found the rate of change in cell area constriction and per pixel myosin intensity buildup rate by finding the difference between a value at a given time step and the value 2 time steps prior, then dividing by the time difference and including only the timepoints between constriction initiation and mesoderm invagination. Finally, we used the MATLAB 'xcorr' function to calculate the normalized cross-correlation between these two rates. We aggregated cross-correlation curves from multiple cells across three embryos from each condition (DMSO- or taxol-injected) and obtained the average cross-correlation plot. 
For the myosin and Patronin pulse analysis, we manually identified 5 myosin pulses in each of the 4 embryos via FIJI and drew an elliptical ROI around each pulse. We recorded the maximum pixel intensity in manually identified ROls for both. To plot the average relative behavior of the Myo::mCherry and Patronin::GFP signals (Fig. $3 \mathrm{C}$ ), we smoothed the intensity data for each pulse by a moving average ( 3 time steps wide) and aligned them such that peak myosin intensity for all pulses would fall at a relative time offset of 0 seconds. Then, we calculated average Myo::mCherry and Patronin::GFP intensities for each relative time offset. We found the normalized crosscorrelation between the rates of change in myosin intensity and Patronin intensity (Fig. $3 \mathrm{E})$ as indicated above.

\section{Immunoblotting}

Early gastrula stage embryos were collected and homogenized in SDS sample buffer, boiled for 5 minutes, and kept on ice. Samples were run on Mini-PROTEAN TGX Precast Gels (Bio-Rad). Primary antibodies used for immunoblotting included $\alpha$-Tubulin (1:500; Sigma-Aldrich) and Patronin (antibody serum; 1:50; a gift from Ron Vale). Primary antibodies were detected by horseradish peroxidase-labeled secondary antibodies. Signal was developed using SuperSignal West Femto Maximum Sensitity Substrate (ThermoFisher).

\section{Drug injections}

Dechorionated embryos were mounted onto glass slides and desiccated for 4 minutes using Drierite (Drierite). Embryos were covered with a 3:1 mixture of halocarbon $700 /$ halocarbon 27 oils and then injected laterally during mid-late cellularization. For ROCK inhibition, Y-27632 was dissolved in water and injected at $50 \mathrm{mM}$ concentration. Seventeen embryos were imaged and quantifications in Fig. $4 \mathrm{~B}$ are from 4 representative embryos. As a control, water was injected. For RhoA inhibition, C3 exoenzyme protein (CT03; Cytoskeleton, Inc.) was resuspended and dialyzed into PBS and injected at $1 \mathrm{mg} / \mathrm{mL}$. Fifteen embryos were imaged and quantifications in Fig. 4 D are from 4 representative embryos. PBS was injected as a control. To depolymerize Factin we resuspended CtyoD (Enzo Life Sciences) at either $5 \mathrm{mg} / \mathrm{mL}$ or $.125 \mathrm{mg} / \mathrm{mL}$ in DMSO. Colchicine and taxol were both resuspended at $5 \mathrm{mg} / \mathrm{mL}$ in DMSO. Embryos were imaged 3-5 minutes after injection. For live injection of colchicine, embryos were mounted ventral side down onto a No.1 Coverslip. The embryo was pierced with the needle on the confocal microscope and injected after tissue folding had initiated during live imaging. 


\section{Acknowledgments}

We would like to thank members of the Martin lab for their helpful comments and suggestions on the project and the manuscript. We thank Frank Mason and lain Cheeseman for their helpful comments on a draft of this manuscript. We would also like to thank Jordan Raff (University of Oxford), Ron Vale (UCSF), Mark Biggin (Berkeley Lab), the Bloomington Stock Center, and TRiP at Harvard Medical School (National Institutes of Health/National Institute of General Medical Sciences R01-GM084947) for fly stocks and antibodies used in this study. This work was supported by grant R01GM105984 to A. C. Martin from the National Institute of General Medical Sciences. The authors declare no competing financial interests.

Author contributions: C. S. Ko and A. C. Martin conceptualized the project and designed experiments. C. S. Ko and A. C. Martin performed the experiments. C. S. Ko and V. Tserunyan analyzed the data. C. S. Ko and A. C. Martin wrote the manuscript. All authors reviewed and approved the final version of the manuscript. 


\section{References}

Agarwal, P., and R. Zaidel-Bar. 2018. Principles of Actomyosin Regulation In Vivo. Trends in Cell Biology. 29(2): 150-163.

Amano, M., M. Ito, K. Kimura, Y. Fukata, K. Chihara, T. Nakano, Y. Matsuura, and K. Kaibuchi. 1996. Phosphorylation and activation of myosin by rho- associated kinase (Rho-kinase). J. Biol. Chem. 271(34): 20246-20249.

Applewhite, D. A., K. D. Grode, D. Keller, A. Zadeh, K. C. Slep, and S. L. Rogers. 2010. The Spectraplakin Short Stop Is an Actin-Microtubule Cross-Linker That Contributes to Organization of the Microtubule Network. Mol. Biol. Cell. 21(10): 1714-1724.

Bacallao, R., C. Antony, C. Dotti, E. Karsenti, E. H. Stelzer, and K. Simons. 1989. The subcellular organization of Madin-Darby canine kidney cells during the formation of a polarized epithelium. J. Cell Biol. 109: 2817-2832.

Barrett, K., M. Leptin, and J. Settleman. 1997. The Rho GTPase and a Putative RhoGEF Mediate a Signaling Pathway for the Cell Shape Changes in Drosophila Gastrulation. Cell. 91(7): 905-915.

Blanchard, G. B., S. Murugesu, R. J. Adams, A. Martinez-Arias, and N. Gorfinkiel. 2010. Cytoskeletal dynamics and supracellular organisation of cell shape fluctuations during dorsal closure. Development. 137(16): 2743-2752.

Booth, A. J. R., G. B. Blanchard, R. J. Adams, and K. Röper. 2014. A Dynamic Microtubule Cytoskeleton Directs Medial Actomyosin Function during Tube Formation. Developmental Cell. 29(5): 562-576.

Chanet, S., R. Sharan, Z. Khan, and A. C. Martin. 2017. Myosin 2-Induced Mitotic Rounding Enables Columnar Epithelial Cells to Interpret Cortical Spindle Positioning Cues. Current Biology. 27(21): 3350-3358.

Chung, S., S. Kim, and D. J. Andrew. 2017. Uncoupling apical constriction from tissue invagination. eLife. doi: 10.7554/eLife.22235.

Colin, A., P. Singaravelu, M. Théry, L. Blanchoin, and Z. Gueroui. (2018). Actin-Network Architecture Regulates Microtubule Dynamics. Current Biology. 28(16): 2647-2656.

Coravos, J. S., and A. C. Martin. 2016. Apical Sarcomere-like Actomyosin Contracts Nonmuscle Drosophila Epithelial Cells. Dev. Cell. 39(3): 346-358.

Costa, M., E. T. Wilson, and E. F. Wieschaus. 1994. A Putative Cell Signal Encoded by the folded gastrulation Gene Coordinates Cell Shape Changes during Drosophila Gastrulation. Cell. 76(6): 1075-1089. 
Crawford, J.M., N. Harden, T. Leung, L. Lim, and D.P. Kiehart. 1998. Cellularization in Drosophila melanogaster is disrupted by the inhibition of rho activity and the activation of Cdc42 function. Dev. Biol. 204(1): 151-164.

David, D. J. V., A. Tishkina, and T. J. C. Harris. 2010. The PAR complex regulates pulsed actomyosin contractions during amnioserosa apical constriction in Drosophila. Development. 137(10): 1645-1655.

Davies, S. P., H. Reddy, M. Caivano, and P. Cohen. 2000. Specificity and mechanism of action of some commonly used protein kinase inhibitors. Biochemical Journal. 351(1): 95-105.

Dawes-Hoang, R. E., K. M. Parmar, A. E. Christiansen, C. B. Phelps, A. H. Brand, and E. F. Wieschaus. 2005. folded gastrulation, cell shape change and the control of myosin localization. Development. 132(18): 4165-4178.

Desai, R., R. Sarpal, N. Ishiyama, M. Pellikka, M. Ikura, and U. Tepass. 2013. Monomeric alpha-catenin links cadherin to the actin cytoskeleton. Nature Cell Biology. 15(3): 261-273.

Fernandez-Gonzalez, R., S. de Matos Simões, J. -C. Röper, S. Eaton, and J. A. Zallen. 2009. Myosin II Dynamics Are Regulated by Tension in Intercalating Cells.

Developmental Cell. 17(5): 736-743.

Fox, D. T. and M. Peifer. 2007. Abelson kinase (Abl) and RhoGEF2 regulate actin organization during cell constriction in Drosophila. Development. 134(3): 567-578.

Gelbart, M. A., B. He, A. C. Martin, S. Y. Thiberge, E. F. Wieschaus, and M. Kaschube. 2012. Volume conservation principle involved in cell lengthening and nucleus movement during tissue morphogenesis. Proceedings of the National Academy of Sciences. 109(47): 19298-19303.

Girdler, G. C., D. A. Applewhite, W. M. G. Perry, S. L. Rogers, and K. Röper. 2016. The Gas2 family protein Pigs is a microtubule +TIP that affects cytoskeleton organisation. J. Cell Sci. 129(1): 121-134.

Goodwin, S. S. and R. D. Vale. 2010. Patronin Regulates the Microtubule Network by Protecting Microtubule Minus Ends. Cell. 143(2): 263-274.

Großhans, J., C. Wenzl, H. -M. Herz, S. Bartoszewski, F. Schnorrer, N. Vogt, H. Schwarz, and H. -Arno Müller. 2005. RhoGEF2 and the formin Dia control the formation of the furrow canal by directed actin assembly during Drosophila cellularisation. Development. 132(5): 1009-1020. 
Häcker, U. and N. Perrimon. 1998. encodes a member of the Dbl family of oncogenes and controls cell shape changes during gastrulation in. Genes \& Development. 12(2): 274-284.

Harris, T. J. C. and M. Peifer. 2005. The positioning and segregation of apical cues during epithelial polarity establishment in Drosophila. J. Cell Biol. 170(5): 813-823.

Harris, T. J. C. and M. Peifer. 2007. aPKC Controls Microtubule Organization to Balance Adherens Junction Symmetry and Planar Polarity during Development. Dev. Cell. 12(5): 727-738.

Heisenberg, C.-P. and Y. Bellaïche. 2013. Forces in Tissue Morphogenesis and Patterning. Cell. 153(5): 948-962.

Hendershott, M. C. and R. D. Vale. 2014. Regulation of microtubule minus-end dynamics by CAMSAPs and Patronin. Proceedings of the National Academy of Sciences. 111(16): 5860-5865.

Henty-Ridilla, J. L., A. Rankova, J. A. Eskin, K. Kenny, and B. L. Goode. 2016. Accelerated actin filament polymerization from microtubule plus ends. Science. 352(6288): 1004-1009.

Jiang, K., S. Hua, R. Mohan, I. Grigoriev, K. W. Yau, Q. Liu, E. A. Katrukha, A. F. Maarten Altelaar, A. J. R. Heck, C. C. Hoogenraad, and A. Akhmanova. 2014. Microtubule Minus-End Stabilization by Polymerization-Driven CAMSAP Deposition. Developmental Cell. 28(3): 295-309.

Jodoin, J. N., J. S. Coravos, S. Chanet, C. G. Vasquez, M. Tworoger, E. R. Kingston, L. A. Perkins, N. Perrimon, and A. C. Martin. 2015. Stable Force Balance between Epithelial Cells Arises from F-Actin Turnover. Developmental Cell. 35(6): 685-697.

Kasza, K. E. and J. A. Zallen. 2011. Dynamics and regulation of contractile actin-myosin networks in morphogenesis. Current Opinion in Cell Biology. 23(1): 30-38.

Khanal, I., A. Elbediwy, M. D. C. Diaz de la Loza, G. C. Fletcher, and B. J. Thompson. 2016. Shot and Patronin polarise microtubules to direct membrane traffic and biogenesis of microvilli in epithelia. J. Cell Sci. 129(13): 2651-2659.

King, M. D. A., G. W. Phillips, P. A. Bignone, N. V. L. Hayes, J. C. Pinder, and A. J. Baines. 2013. A conserved sequence in calmodulin regulated spectrin-associated protein 1 links its interaction with spectrin and calmodulin to neurite outgrowth. Journal of Neurochemistry. 128(3): 391-402.

Kölsch, V., T. C. Seher, G. J. Fernandez-Ballester, L. Serrano, and M. Leptin. 2007. Control of Drosophila Gastrulation by Apical Localization of Adherens Junctions and RhoGEF2. Science. 315(5810) : 384-386. 
Le Droguen, P.-M., S. Claret, A. Guichet, and V. Brodu. 2015. Microtubule-dependent apical restriction of recycling endosomes sustains adherens junctions during morphogenesis of the Drosophila tracheal system. Development. 142(2): 363-374.

Lecuit, T., P. -F. Lenne, and E. M. Munro. (2011). Force generation, transmission, and integration during cell and tissue morphogenesis. Annual Review of Cell and Developmental Biology. 27(1): 157-184.

Lecuit, T. and A. S. Yap. 2015. E-cadherin junctions as active mechanical integrators in tissue dynamics. Nat. Cell Bio. 17(5): 533-539.

Lee, J.-Y. and R. M. Harland. 2007. Actomyosin contractility and microtubules drive apical constriction in Xenopus bottle cells. Developmental Biology. 311(1): 40-52.

Lee, C., H. M. Scherr, and J. B. Wallingford. 2007. Shroom family proteins regulate gamma-tubulin distribution and microtubule architecture during epithelial cell shape change. Development. 134(7): 1431-1441.

Leptin, M. and B. Grunewald. 1990. Cell shape changes during gastrulation in Drosophila. Development. 110(1): 73-84.

López, M. P., F. Huber, I. Grigoriev, M. O. Steinmetz, A. Akhmanova, G. H. Koenderink, and M. Dogterom. 2014. Actin-microtubule coordination at growing microtubule ends. Nature Communications. 5(4778). doi: 10.1038/ncomms5778.

Marston, D. J., C. D. Higgins, K. A. Peters, T. D. Cupp, D. J. Dickinson, A. M. Pani, R. P. Moore, A. H. Cox, D. P. Kiehart, and B. Goldstein 2016. MRCK-1 Drives Apical Constriction in C. elegans by Linking Developmental Patterning to Force Generation. Current Biology. 26(16): 2079-2089.

Martin, A. C., M. Gelbart, R. Fernandez-Gonzalez, M. Kaschube, and E. F. Wieschaus. 2010. Integration of contractile forces during tissue invagination. The Journal of Cell Biology. 188(5): 735-749.

Martin, A. C. and B. Goldstein. 2014. Apical constriction: themes and variations on a cellular mechanism driving morphogenesis. Development. 141(10): 1987-1998.

Mason, F. M., M. Tworoger, and A. C. Martin. 2013. Apical domain polarization localizes actin--myosin activity to drive ratchet-like apical constriction. Nat. Cell Bio. 15(8): 926936.

Mason, F. M., S. Xie, C. G. Vasquez, M. Tworoger, and A. C. Martin. 2016. RhoA GTPase inhibition organizes contraction during epithelial morphogenesis. The Journal of Cell Biology. 214(5): 603-617. 
Meng, W., Y. Mushika, T. Ichii, and M. Takeichi. 2008. Anchorage of Microtubule Minus Ends to Adherens Junctions Regulates Epithelial Cell-Cell Contacts. Cell. 135(5): 948959.

Mizuno, T., M. Amano, K. Kaibuchi, and Y. Nishida. 1999. Identification and characterization of Drosophila homolog of Rho-kinase. Gene. 238(2): 437-444.

Mogensen, M. M., A. Malik, M. Piel, V. Bouckson-Castaing, M. Bornens. 2000.

Microtubule minus-end anchorage at centrosomal and non-centrosomal sites: the role of ninein. J. Cell Sci. 113: 3013-3023.

Moss, D. K., G. Bellett, J. M. Carter, M. Liovic, J. Keynton, A. R. Prescott, E. Birgitte Lane, and M. M. Mogensen. 2007. Ninein is released from the centrosome and moves bi-directionally along microtubules. Journal of Cell Science. 120: 3064-3074.

Murrell, M., P. W. Oakes, M. Lenz, and M. L. Gardel. 2015. Forcing cells into shape: the mechanics of actomyosin contractility. Nature Reviews Molecular Cell Biology. 16(8): 486-498.

Nagae, S., W. Meng, and M. Takeichi. 2013. Non-centrosomal microtubules regulate Factin organization through the suppression of GEF-H1 activity. Genes to Cells. 18(5): 387-396.

Ning, W., Y. Yu, H. Xu, X. Liu, D. Wang, J. Wang, Y. Wang, and W. Meng. 2016. The CAMSAP3-ACF7 Complex Couples Noncentrosomal Microtubules with Actin Filaments to Coordinate Their Dynamics. Dev. Cell. 39(1): 61-74.

Noordstra, I., Q. Liu, W. Nijenhuis, S. Hua, K. Jiang, M. Baars, S. Remmeizwaal, M. Martin, L. C. Kapitein, and A. Akhmanova. 2016. Control of apico-basal epithelial polarity by the microtubule minus-end-binding protein CAMSAP3 and spectraplakin ACF7. J. Cell Sci. 129(22): 4278-4288.

Rogers, S. L., U. Wiedemann, U. Häcker, C. Turck, and R. D. Vale. 2004. Drosophila RhoGEF2 Associates with Microtubule Plus Ends in an EB1-Dependent Manner. Current Biology. 14(20): 1827-1833.

Roh-Johnson, M., G. Shemer, C. D. Higgins, J. H. McClellan, A. D. Werts, U. S. Tulu, L. Gao, E. Betzig, D. P. Kiehart, and B. Goldstein. 2012. Triggering a Cell Shape Change by Exploiting Preexisting Actomyosin Contractions. Science. 355: 1232-1235.

Royou, A., W. Sullivan, and R. Karess. 2002. Cortical recruitment of nonmuscle myosin II in early syncytial Drosophilaembryos. The Journal of Cell Biology. 158(1): 127-137.

Royou, A., C. Field, J. C. Sisson, W. Sullivan, and R. Karess. 2004. Reassessing the Role and Dynamics of Nonmuscle Myosin II during Furrow Formation in Early Drosophila Embryos. Mol. Biol. Cell. 15(2): 838-850. 
Salmon, W. C., M. C. Adams, and C. M. Waterman-Storer. 2002. Dual-wavelength fluorescent speckle microscopy reveals coupling of microtubule and actin movements in migrating cells. The Journal of Cell Biology. 158(1): 31-37.

Sánchez-Corrales, Y. E., G. B. Blanchard, and K. Röper. 2018. Radially patterned cell behaviours during tube budding from an epithelium. eLife. doi: 10.7554/eLife.35717.

Sawyer, J. K., N. J. Harris, K. C. Slep, U. Gaul, and M. Peifer. 2009. The Drosophila afadin homologue Canoe regulates linkage of the actin cytoskeleton to adherens junctions during apical constriction. The Journal of Cell Biology. 186(1): 57-73.

Sawyer, J. M., J. R. Harrell, G. Shemer, J. Sullivan-Brown, M. Roh-Johnson, and B. Goldstein. 2010. Apical constriction: A cell shape change that can drive morphogenesis. Developmental Biology. 341(1): 5-19.

Sawyer, J. K., W. Choi, K. -C. Jung, L. He, N. J. Harris, and M. Peifer. 2011. A contractile actomyosin network linked to adherens junctions by Canoe/afadin helps drive convergent extension. Molecular Biology of the Cell. 22(14): 2423-2659.

Simões, S., B. Denholm, D. Azevedo, S. Sotillos, P. Martin, H. Skaer, J. C. G. Hombría, and A. Jacinto. 2006. Compartmentalisation of Rho regulators directs cell invagination during tissue morphogenesis. Development. 133(21): 4257-4267.

Singh, A., T. Saha, I. Begemann, A. Ricker, H. N. X. Nüsse, O. Thorn-Seshold, J. Klingauf, M. Galic, and M. Matis. 2018. Polarized microtubule dynamics directs cell mechanics and coordinates forces during epithelial morphogenesis. Nature Cell Biology. 20: 1126-1133.

Stehbens, S. J., A. D. Paterson, M. S. Crampton, A. M. Shewan, C. Ferguson, A. Akhmanova, R. G. Paton, and A. S. Yap. 2006. Dynamic microtubules regulate the local concentration of E-cadherin at cell-cell contacts. Journal of Cell Science. 119: 18011811.

Sweeton, D., S. Parks, M. Costa, and E. F. Wieschaus. 1991. Gastrulation in Drosophila: the formation of the ventral furrow and posterior midgut invaginations. Development. 112(3): 775-789.

Takács, Z., F. Jankovics, P. Vilmos, P. Lénárt, K. Röper, and M. Erdélyi. 2017. The spectraplakin short stop is an essential microtubule regulator involved in epithelial closure in Drosophila. J. Cell Sci. 130(4): 712-724.

Takeda, M., M. M. Sami, and Y. C. Wang. 2018. A homeostatic apical microtubule network shortens cells for epithelial folding via a basal polarity shift. Nat. Cell Bio. 20(1): 36-45. 
Tanaka, N., W. Meng, S. Nagae, and M. Takeichi. 2012. Nezha/CAMSAP3 and CAMSAP2 cooperate in epithelial-specific organization of noncentrosomal microtubules. Proceedings of the National Academy of Sciences. 109(49): 20029-20034.

Toya, M., S. Kobayashi, M. Kawasaki, G. Shioi, M. Kaneko, T. Ishiuchi, K. Misaki, W. Meng, and M. Takeichi. 2016. CAMSAP3 orients the apical-to-basal polarity of microtubule arrays in epithelial cells. Proceedings of the National Academy of Sciences. 113(2): 332-337.

Vasquez, C. G., M. Tworoger, and A. C. Martin. 2014. Dynamic myosin phosphorylation regulates contractile pulses and tissue integrity during epithelial morphogenesis. The Journal of Cell Biology. 206(3): 435-450.

Weng, M. and E. F. Wieschaus. 2016. Myosin-dependent remodeling of adherens junctions protects junctions from Snail-dependent disassembly. The Journal of Cell Biology. 212(2): 219-229.

Westermann, S. and K. Weber. 2003. Post-translational modifications regulate microtubule function. Nature Reviews Molecular Cell Biology. 4(12): 938-948.

Wu, S. Z. and M. Bezanilla. 2018. Actin and microtubule cross talk mediates persistent polarized growth. J. Cell Biol. 217(10): 3531-3544.

Xie, S., F. M. Mason, and A. C. Martin. 2016. Loss of $\mathrm{G}_{a 12 / 13}$ exacerbates apical area dependence of actomyosin contractility. Mol. Biol. Cell. 27(22): 3526-3536. 
Figure 1. Patronin::GFP localizes medioapically in apically constricting cells.

(A) Diagram of an embryo undergoing mesoderm invagination. Ventral, mesoderm cells (snail expressing domain highlighted in red) apically constrict and internalize, forming a ventral furrow along the midline (dashed line). (B) Patronin::GFP is present in a medioapical focus specifically in the mesoderm (top row, yellow arrowhead).

Patronin::GFP is enriched at junctions in the ectoderm (bottom row, white arrowhead). Images are maximum intensity projections from a live embryo expressing Patronin::GFP (apical surface) and Gap43::mCH (plasma membranes, sub-apical slice). (C)

Patronin::GFP localization changes from junctional (white arrowheads) to medioapical (yellow arrowheads) in the mesoderm. Images are apical-basal cross-sections from a live embryo expressing Patronin::GFP and Gap43::mCH. Top row: mid-cellularization; middle row: late-cellularization/early gastrulation; bottom row: during folding. Nuclei are highlighted by dashed white lines. (D) Quantification of medioapical Patronin::GFP enrichment. Individual cells were segmented and the junctional and medioapical Patronin::GFP intensity was calculated and the distribution of the ratio (junctional/medioapical) plotted ( $\mathrm{n}=6$ embryos, 559 cells; ${ }^{* *}, \mathrm{P}<.0001$, KolmogorovSmirnov test). (E) Apical Patronin::GFP foci are more intense in the mesoderm than the ectoderm. The maximum apical Patronin::GFP intensity was determined in a region of the embryo ( $n=3$ embryos, 10 measurements per embryo; ${ }^{* *}, P<.0001$, unpaired $t$ test). Notch is the median, bottom and top edges of the box are the $25^{\text {th }}$ and $75^{\text {th }}$ percentiles, whiskers extend to the most extreme data points. (F) Medioapical Patronin::GFP co-localizes with apical myosin patches. Images are apical surface Zprojections from a live embryo expressing Patronin::GFP and Myo::mCH (sqh::mCH). 
(G) Patronin::GFP localizes medioapically in apically constricting endoderm cells.

Images are maximum intensity projections of a fixed embryo expressing Patronin::GFP

(apical surface). The embryo was immunostained with Phalloidin conjugated with AF647

to visualize cell outlines (sub-apical section). Scale bars, $10 \mu \mathrm{m}$ (G, top) and $5 \mu \mathrm{m}$ (A-

G, bottom).

Figure 2. Patronin stabilizes apical, non-centrosomal microtubules in the

mesoderm. (A) In the mesoderm, microtubules are oriented parallel and perpendicular to the apical membrane. Images are maximum intensity projections from a fixed embryo stained for $\alpha$-Tubulin (apical surface) and F-actin (sub-apical section, phalloidin). Surface views of cells along the midline and at the edge of the snail-expressing domain are shown for an embryo at folding initiation and mid-folding. (B) Apical microtubules are enriched in mesoderm. Image is apical surface Z-projection of a fixed embryo stained for $\alpha$-Tubulin. The furrow midline is marked with a dashed white line. (C) GFP::CLIP170 form dense clusters at the apical surface, many of which co-localize with myosin (yellow circles). Images are single apical sections from a live movie of an embryo expressing GFP::CLIP170 and Myo::mCH (sqh::mCH). (D) Depleting Patronin reduces apical microtubules. Images are apical surface projections of fixed embryos stained for acetylated-Tubulin. The surface view of control RNAi knockdown (top) and Patronin RNAi knockdown (bottom) are shown on the left. The ventral midline is marked by a white dashed line. The right set of images show cross-sections of the same embryos on the left. The left image is a single section and the right image is a maximum intensity projection of 10 cross-sections. (E). Patronin::GFP localization depends on an 
intact microtubule cytoskeleton. Images are a montage from a live movie of an embryo expressing Patronin::GFP and injected with either DMSO (top) or Colchicine (5 mg/mL; bottom). Scale bars, $5 \mu \mathrm{m}$.

\section{Figure 3. Medioapical Patronin foci form by coalescence during myosin}

assembly. (A) Patronin foci form by coalescence of smaller puncta (arrowheads). Timelapse images are from a single apical section from a live movie of an embryo expressing Patronin::GFP. (B) Patronin puncta coalescence (arrowheads) occurs during myosin pulse assembly. Time-lapse images represent a myosin pulse (single apical slice) from a live movie of an embryo expressing Patronin::GFP and Myo::mCH. (C) Quantification of maximum normalized Myo::mCH and Patronin::GFP intensity $(\mathrm{n}=4$ embryos, 5 pulses per embryo) that has been averaged by aligning the maxima of Myo::mCH across all 20 pulses. Time at peak mean myosin intensity was centered at 0. Error bars represent the standard error of the mean. (D) During myosin pulse disassembly, local Patronin::GFP intensity decreases and larger foci break apart into smaller puncta (arrowheads). Time-lapse images are a representative pulse from a single apical section from a live movie of an embryo expressing Patronin::GFP and Myo::mCH. (E) Myosin and Patronin intensity are tightly correlated. Plotted is the mean time-resolved correlation function between changes in $\mathrm{Myo::mCH}$ and Patronin::GFP intensity. Maximum correlation occurs at an offset of $\sim-4$ seconds. Dashed line indicates 0 offset. Scale bars, $3 \mu \mathrm{m}$. 
Figure 4. Medioapical Patronin foci depend on actomyosin contraction. (A) ROCK inhibitor disrupts apical Patronin localization in the mesoderm. Images are maximum intensity Z-projections of mesoderm cells from live embryos expressing Patronin::GFP (apical surface) and Gap43::mCH (sub-apical section) injected with water (top) or Y27632 (50 mM; bottom). (B) ROCK inhibitor decreases Patronin::GFP apical intensity. Quantification of maximum Patronin::GFP intensity in a region that spans the apical cortex $\left(n=7\right.$ measurements per embryo, 4 embryos per condition; ${ }^{* *}, P<.0001$, unpaired t test). Error bars represent one standard deviation. (C) RhoA inhibition disrupts medioapical Patronin localization. Images are maximum intensity Z-projections from a live embryo expressing Patronin::GFP (apical surface) and Gap43::mCH (subapical section) injected with PBS (top) or the C3 exoenzyme (1 mg/mL; bottom). (D) RhoA inhibition decreases apical Patronin::GFP intensity. Quantification of maximum Patronin::GFP intensity in a region that spans the apical cortex $(n=7$ measurements per embryo, 4 embryos per condition; ${ }^{* *}, \mathrm{P}<.0001$, unpaired $\mathrm{t}$ test). Error bars represent one standard deviation. (E) Disrupting the apical F-actin network disrupts medioapical Patronin foci. Time-lapse images are maximum intensity Z-projections from live embryos expressing Patronin::GFP (apical surface) and Gap43::mCH (sub-apical section) injected with DMSO (left) and CytoD (.125 mg/mL; right). Scale bars, $5 \mu \mathrm{m}$.

Figure 5. Apical myosin activation and apical constriction initiation do not require microtubules. (A) Disrupting microtubules disrupts folding, despite apical myosin activation and apical constriction initiation. Time-lapse images are maximum intensity Zprojections from live embryos expressing sqh::GFP (myosin, apical surface) and 
Gap43::mCH (membrane, sub-apical section) injected with DMSO (left), colchicine (5 $\mathrm{mg} / \mathrm{mL}$; middle), and taxol (5 mg/mL; right). Apical-basal cross-sections are to the right of each image. Dashed line indicates the ventral furrow. (B) Apical area initiates reduction after disrupting microtubules. Quantification of apical cell areas preconstriction $(t=0 \mathrm{~s})$ and $320 \mathrm{~s}$ after from three representative live embryos injected with DMSO ( $n=226$ cells, $T=0$ s; 252 cells, $T=320 \mathrm{~s} ;{ }^{* *}, P<.0001$, unpaired t test), colchicine $\left(n=284\right.$ cells, $T=0$ s; 353 cells, $T=320 \mathrm{~s} ;{ }^{* *}, P<.0001$, unpaired $t$ test $)$, and taxol $\left(\mathrm{n}=275\right.$ cells, $\mathrm{T}=0 \mathrm{~s} ; 226$ cells, $\mathrm{T}=320 \mathrm{~s} ;{ }^{* *}, \mathrm{P}<.0001$, unpaired $\mathrm{t}$ test). Notch is the median, bottom and top edges of the box are the $25^{\text {th }}$ and $75^{\text {th }}$ percentiles, whiskers extend to the most extreme data points. (C) Myosin assembly and pulses still occur after inhibiting microtubule dynamics. Graphs show apical area and myosin intensity over time in representative single cells in DMSO- (top) and taxol-injected (bottom) embryos. (D) Disrupting microtubule dynamics does not initially interfere with the correlation between myosin increase and area reduction. Plotted is the mean timeresolved correlation function between constriction rate $(+$ constriction $=$ area decrease $)$ and myosin rate. Data are from 3 representative embryos injected with DMSO ( $\mathrm{n}=548$ cells) or taxol ( $n=543$ cells). Error bars represent the standard error of the mean. $(E)$ Microtubule disruption does not affect apical ROCK polarity. Images show apical-basal cross-sections from representative live embryos expressing ROCK::GFP and Gap43mCH and injected with DMSO, colchicine, or taxol. (F) Apical spot adherens junctions are unaffected by microtubules disruption. Images are apical surface projections of a live embryo expressing E-cadherin::GFP and Myo::mCH injected with 
DMSO (left), colchicine (middle), and taxol (right). Cross-sections are shown on the bottom. Scale bars, $15 \mu \mathrm{m}(\mathbf{A}), 10 \mu \mathrm{m}(\mathbf{E})$ and $5 \mu \mathrm{m}(\mathbf{F})$.

\section{Figure 6. Microtubules stabilize actomyosin connections to intercellular}

junctions. (A) Microtubule disruption leads to separations between myosin structures and intercellular junctions. Time-lapse images are maximum intensity Z-projections from live embryos expressing Myo::GFP (apical surface) and Gap43::mCH (sub-apical section illustrating junctions) injected with DMSO (top), colchicine ( $5 \mathrm{mg} / \mathrm{mL}$; middle), and taxol (5 mg/mL; bottom). Red arrows indicate the direction in which myosin structures separate from cell junctions. (B) Myosin separation events are dynamic and attachments are reestablished following separation. Time-lapse images are maximum intensity Z-projections of apical Myo::GFP in embryos injected with colchicine or taxol. Individual myosin patches are labeled with colored dots. Fiber-like structures between myosin patches that either break or re-form are shown by white arrowheads. (C) Taxol does not affect ROCK polarity, but causes separation between ROCK foci and junctions. Time-lapse images are maximum intensity Z-projections from live embryos expressing GFP::ROCK and Gap43::mCH injected with DMSO or taxol (5 mg/mL). Red arrows indicate the direction in which ROCK foci separate from cell junctions. (D) Microtubules are continuously required during tissue folding. Microtubules were acutely inhibited by injecting embryo with colchicine $\sim 20$ seconds after start of imaging. Images are maximum intensity Z-projections from a live embryo expressing Myo::GFP and Gap43::mCH. (E) Depleting Patronin also causes myosin network separation. Time-lapse images are apical projections from representative live embryos expressing 
Myo::GFP and shRNA against Rhodopsin-3 (control, top) and Patronin (bottom). Red arrows indicate myosin structures separating. (F) Diagram showing model for myosin separation. Shown is a top down view of the apical cortex in two adjacent cells. Unstable attachment of actomyosin to the adherens junction (blue dot) after microtubule disruption leads to separation. Scale bars, $5 \mu \mathrm{m}$.

\section{Figure 7. Actin and microtubule cytoskeletons interact to promote stable}

intercellular force transmission. (A) Diagrams at top show the proposed organization of the microtubule and actin cytoskeletal networks at the cell apex. Diagrams below show top down views of the apical cortex and the effect of various perturbations on Rho/ROCK/F-actin and Patronin localization. C3 injection eliminates apical RhoA/ROCK and Patronin foci. CytoD/LatB injections lead to formation of smaller RhoA/ROCK and Patronin puncta. Colchicine injection does not affect RhoA/ROCK polarity but leads to actomyosin network separations from intercellular junctions. (B) Patronin puncta colocalize with myosin puncta after F-actin network fragmentation with LatB (yellow arrowheads). Time-lapse images are maximum intensity Z-projections of embryos expressing and Patronin::GFP and Myo::mCH injected with LatB (5 mg/mL in DMSO). Scale bars, $5 \mu \mathrm{m}$. 
Movie 1. Patronin::GFP forms a medioapical focus in mesoderm cells. Embryos expressing Patronin::GFP (green) and Gap43::mCH (magenta) shown with the midline of the mesoderm centered (top) or slightly turned (bottom) to show the ectoderm. Images were acquired every 44 seconds (top) or 40 seconds (bottom) and videos are displayed at 10 frames per second. Bars, $15 \mu \mathrm{m}$.

Movie 2. GFP::CLIP170 puncta co-localize with apical myosin. Embryo expressing GFP::CLIP170 (green) and Myo::mCH (magenta). Images were acquired every 1.9 seconds and video is displayed at 15 frames per second. Bars, $15 \mu \mathrm{m}$.

\section{Movie 3. Patronin medioapical foci in mesoderm cells form by myosin} contraction. Embryo expressing Patronin::GFP (green) and Myo::mCH (magenta). Images were acquired every 1.9 seconds and video is displayed at 15 frames per second. Bars, $15 \mu \mathrm{m}$.

\section{Movie 4. Colchicine and taxol disrupt mesoderm invagination but does not interfere with apical constriction initiation. Embryos expressing Myo::GFP (green) and Gap43::mCH (magenta) were injected with DMSO (top), colchicine ( $5 \mathrm{mg} / \mathrm{mL}$; middle), or taxol (5 mg/mL; bottom). Images were acquired every 6.4 seconds and videos are displayed at 15 frames per second. Bars, $15 \mu \mathrm{m}$.}

\section{Movie 5. Microtubule disruption destabilizes intercellular actomyosin} attachments. Embryo expressing Myo::GFP was injected with colchicine $(5 \mathrm{mg} / \mathrm{mL}$; top). Images were acquired every 10.4 seconds (top) or 24 seconds (bottom) and videos are displayed at 15 frames per second. Bar, $15 \mu \mathrm{m}$. 


\section{Supplemental Material}

Figure S1. Medioapical Patronin foci are not centrosomes. (A) Time-lapse images showing an apical-basal cross-section from a live embryo expressing DTACC::GFP (green, a marker for centrosomes; Gergely et al., 2000) and Gap43::mCH (magenta). A kymograph using a line drawn along the apical-basal axis of a cell at the midline is shown on the right. (B) Apical Patronin foci do not co-localize with centrosome markers. Images are maximum intensity projections of apical-basal cross-sections from a fixed embryo stained for $\gamma$-Tubulin (associated with centrosomes; Dictenberg et al., 1998) (red), Asterless (Cep152, a centriolar component; Varmark et al., 2007; Dzhindzhev et al., 2010) (blue), and endogenous Patronin::GFP signal (green). Medioapical Patronin (yellow arrowhead) is separate from Patronin::GFP signal at centrosomes (white arrowhead) (C) Lysates from control-shRNA and Patronin-shRNA flies probed with Patronin antibody serum. Tubulin was used as a loading control. Scale bars, $5 \mu \mathrm{m}$.

Figure S2. CytoD and LatB affect the organization of apical microtubules. (A) Disruption of actin leads to formation of microtubule bundles that lay parallel to the apical surface. Time-lapse images are maximum intensity Z-projections from live embryos expressing GFP::CLIP170 and Myo::mCH injected with either a low dose of CytoD (125 mg/mL; left) or high dose (5 mg/mL; right). (B) Patronin foci fragment into puncta after LatB injection. Time-lapse images are maximum intensity Z-projections of a representative embryo expressing Patronin::GFP and Gap43:mCH injected with Latrunculin B (5 mg/mL in DMSO). Scale bars, $10 \mu \mathrm{m}$ (A), $5 \mu \mathrm{m}$ (B).

Figure S3. Patronin depletion disrupts folding, but not apical adherens junctions. (A) Depleting Patronin disrupts folding, despite apical myosin activation and apical constriction initiation. Time-lapse images are maximum intensity Z-projections from live embryos expressing control-shRNA (left) or Patronin-shRNA (right) and Myo::GFP (apical surface) and Gap43::mCH (sub-apical section). The phenotype was observed in 5 out of 12 embryos imaged from this cross. (B) In mesoderm cells, adherens junctions exhibit apical shift after Patronin depletion. Images are apical-basal cross-sections from embryos fixed at different developmental stages stained for $\beta$-catenin (Armadillo) and 
Snail. (C) Apical adherens junctions are unaffected by Patronin depletion. Time-lapse images show apical-basal cross sections from representative live embryos expressing control-shRNA (left) or Patronin-shRNA (right) and E-cadherin::GFP and $\mathrm{Myo}:: \mathrm{mCH}$.

Thirty-two embryos were imaged in total. (D) Taxol injection causes dense microtubule bundles across the apical surface. Time-lapse images are maximum intensity Zprojections from live embryos expressing GFP::CLIP170 (apical surface) and Gap43::mCH (sub-apical section) injected with either DMSO (top) or taxol ( $5 \mathrm{mg} / \mathrm{mL}$; bottom). Scale bars, $10 \mu \mathrm{m}$ (A, C, D), $5 \mu \mathrm{m}$ (B).

\section{Figure S4. Disrupting microtubules cause actomyosin separation from adherens} junctions. (A) E-cadherin is still present at interfaces between myosin spot separations. Time-lapse images are maximum intensity Z-projections of apical $\mathrm{Myo::mCH}$ and an apical Z-slice of E-cadherin::GFP for a representative embryo injected with taxol (5 $\mathrm{mg} / \mathrm{mL}$ ). Red arrows indicate the direction in which myosin spots separate from cell junctions. One cell-cell interface is highlighted between yellow arrowheads. (B) Ecadherin eventually loses junctional polarity over time. Time-lapse images showing a single apical (cyan) and sub-apical (red) slice of an embryo expressing E-cadherin::GFP injected with colchicine (left) and taxol (right). Scale bars, $10 \mu \mathrm{m}(\mathbf{A}), 5 \mu \mathrm{m}(\mathbf{B})$.

\section{Supplemental References}

Dictenberg, J. B., W. Zimmerman, C. A. Sparks, A. Young, C. Vidair, Y. Zheng, W. Carrington, F. Fay, and S. J. Doxsey. 1998. Pericentrin and y-Tubulin Form a Protein Complex and Are Organized into a Novel Lattice at the Centrosome. J. Cell Biol. 141(1): 163174.

Dzhindzhev, N. S., Q. D. Yu, K. Weiskopf, G. Tzolovsky, I. Cunha-Ferreira, M. Riparbelli, A. Rodrigues-Martins, M. Bettencourt-Dias, G. Callaini, and D. M. Glover. 2010. Asterless is a scaffold for the onset of centriole assembly. Nature. 467: 714-718.

Gergely, F., D. Kidd, K. Jeffers, J. G. Wakefield, and J. W. Raff. 2000. D-TACC: a novel centrosomal protein required for normal spindle function in the early Drosophila embryo. The EMBO Journal. 19(2): 241-252.

Varmark, H., S. Llamazares, E. Rebollo, B. Lange, J. Reina, H. Schwarz, and C. Gonzalez. 2007. Asterless Is a Centriolar Protein Required for Centrosome Function and Embryo Development in Drosophila. Curr. Bio. 17(20): 1735-1745. 
pioRxiv preprint doi: https://doi.org/10.1101/540344; this version posted February 4, 2019. The copyright holder for this preprint (which was not certified by peer review) is the author/funder, who has granted bioRxiv a license to display the preprint in perpetuity. It is made available under

A

midline ectoderm

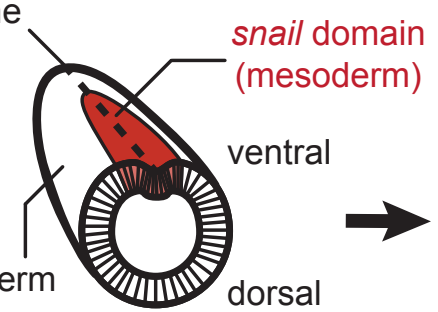
aCC-BY-NC-ND 4.0 International license.

E

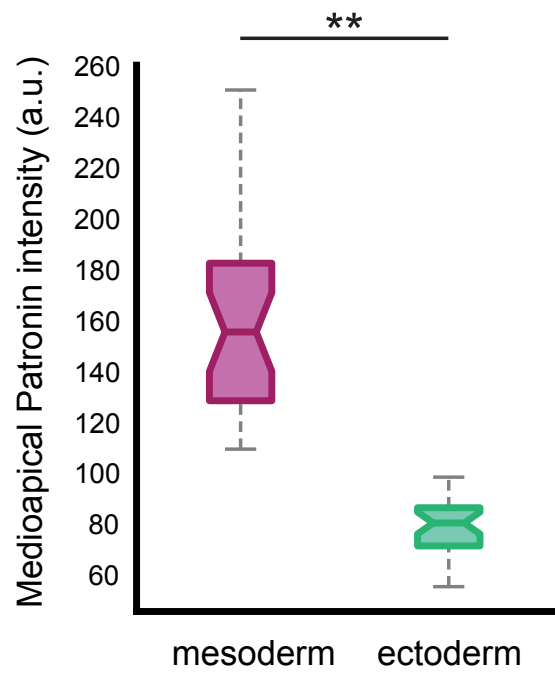

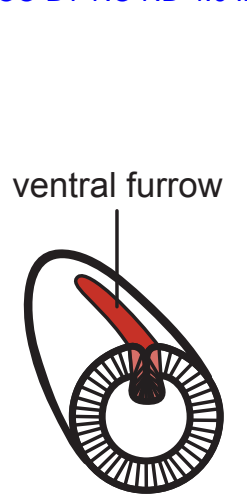

B

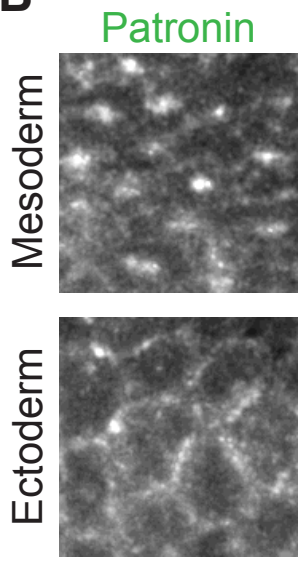

D
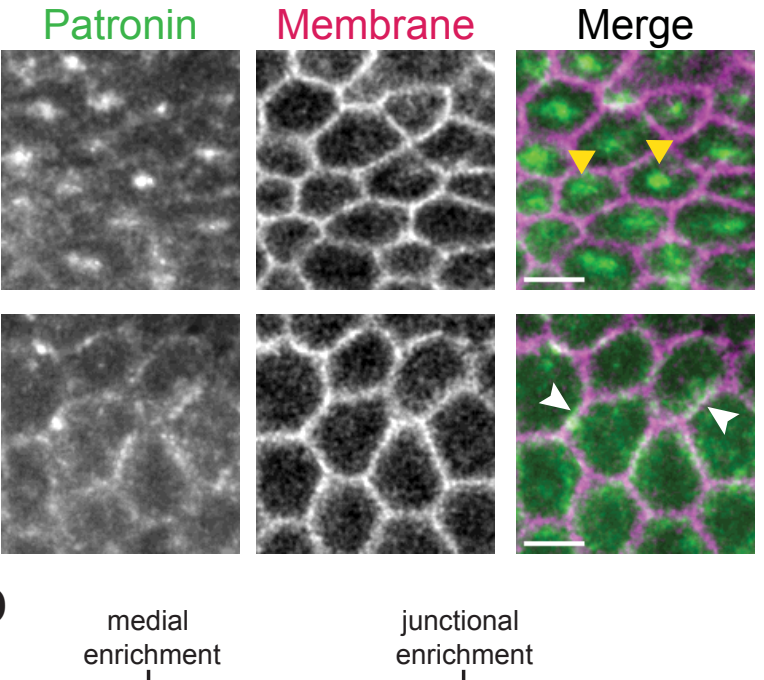

Ratio of avg. junctional over medial Patronin intensity
$\mathbf{F}$

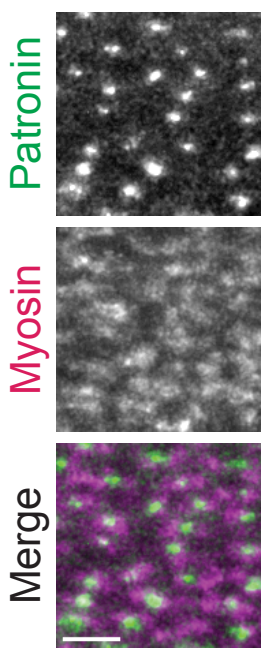

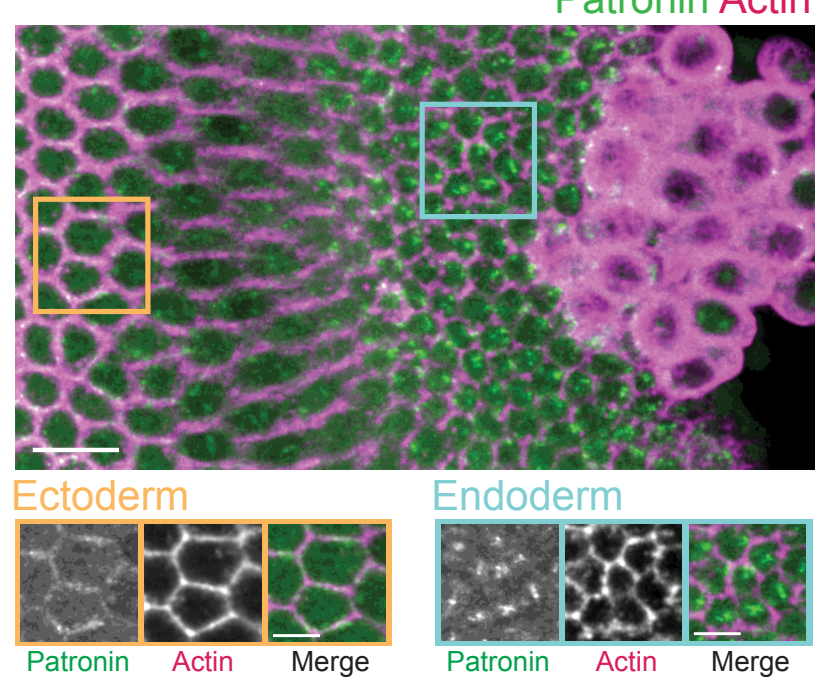
Patronin Actin

Patronin

Actin

Patronin

Merge
G

- Mesoderm

— Ectoderm basal
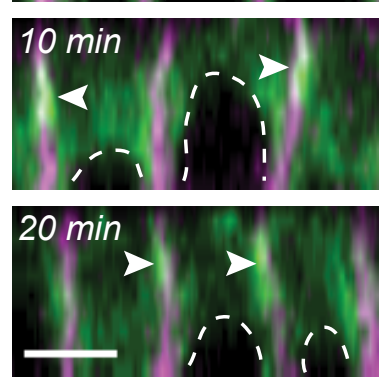
Figure bioRxiv preprint doi: https://doi.org/10.1101/540344; this version posted February 4, 2019. The copyright holder for this preprint (which was not Eertified by peer review) is the author/funder, who has granted bioRxiv a license to display the preprint in perpetuity. It is made available under

A folding initiation

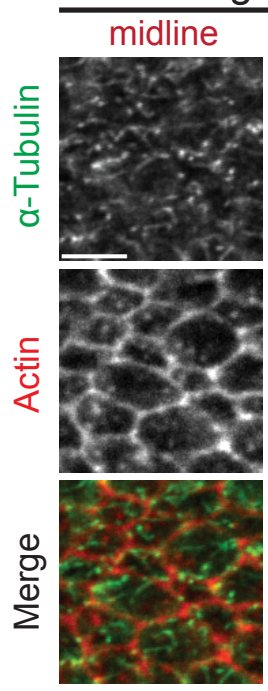

D

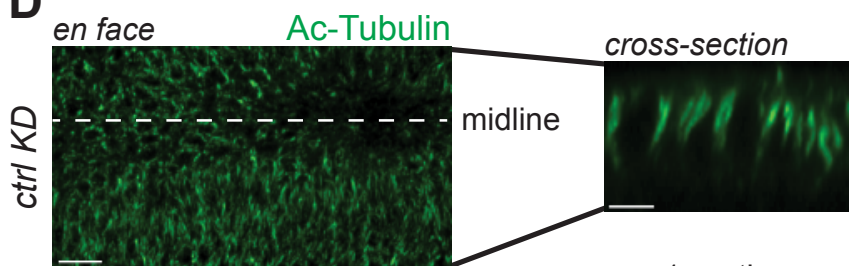

率
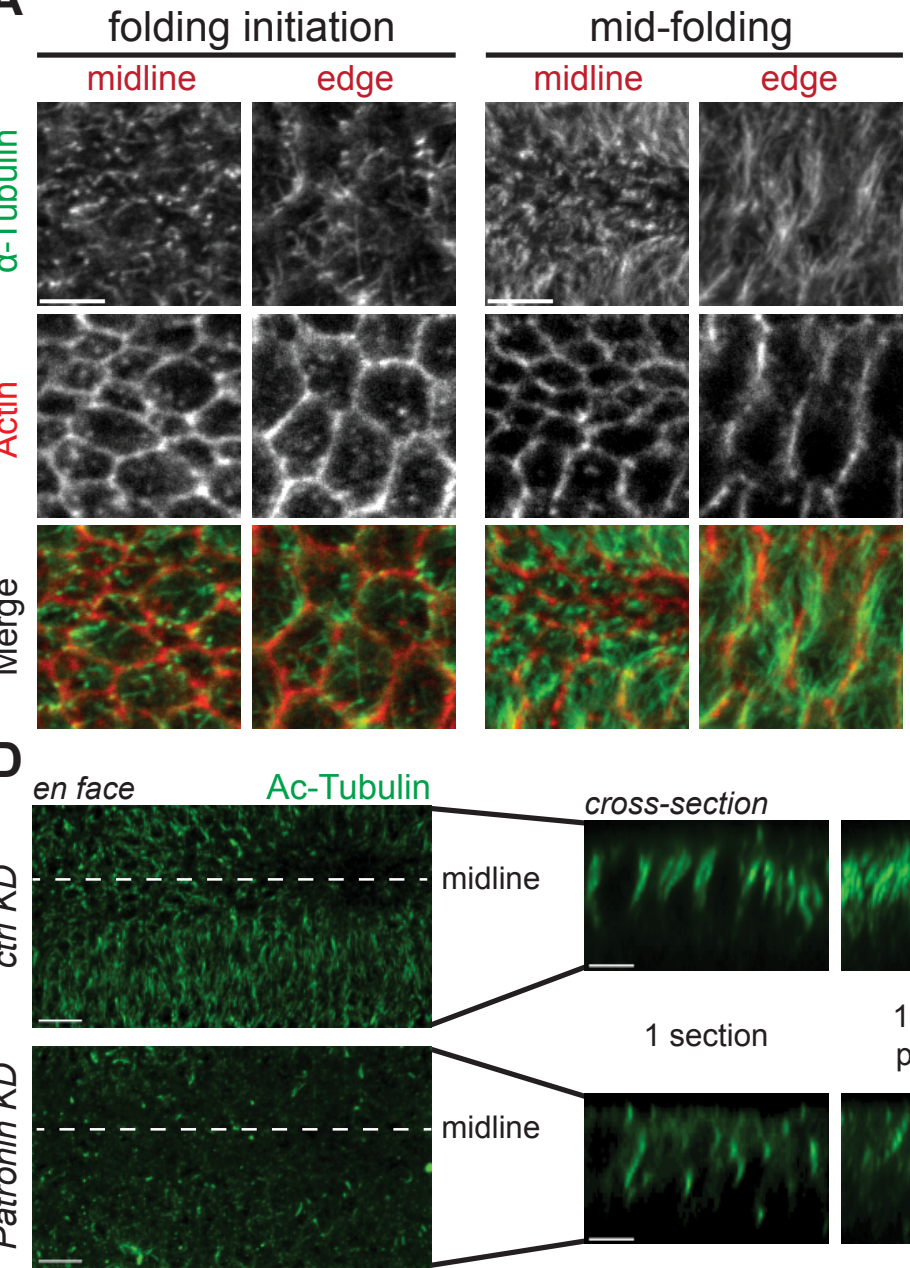

B

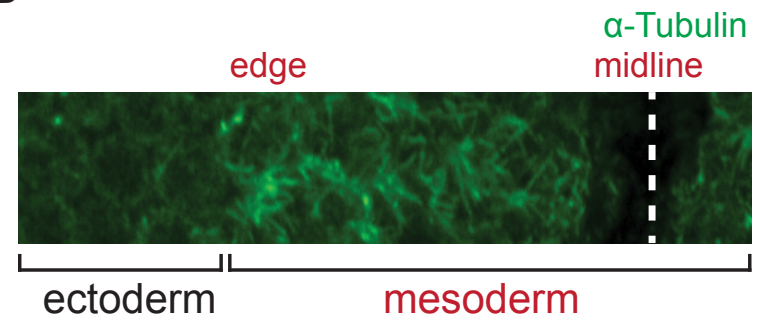

C

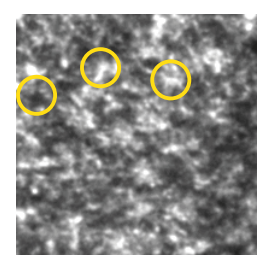

CLIP170

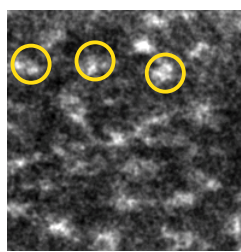

Myosin

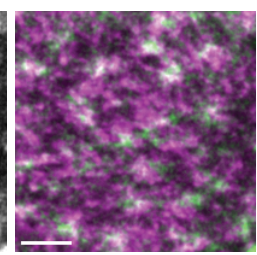

Merge

E

10 section projection

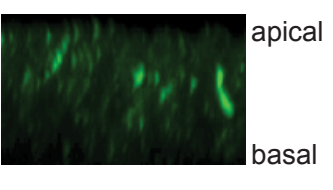

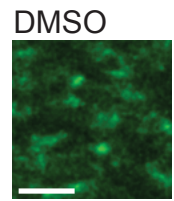

0 min

Colchicine

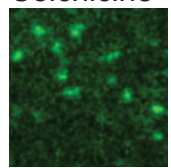

0 min

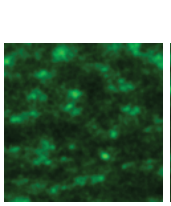

1 min

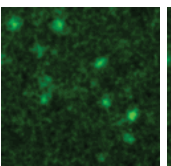

1 min
Patronin

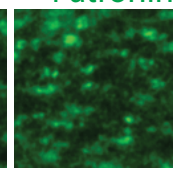

2 min

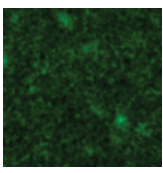

$2 \min$ 
Fing dioRxiv preprint doi: https://doi.org/10.1101/540344; this version posted February 4, 2019. The copyright holder for this preprint (which was not 3 certified by peer review) is the author/funder, who has granted bioRxiv a license to display the preprint in perpetuity. It is made available under

A

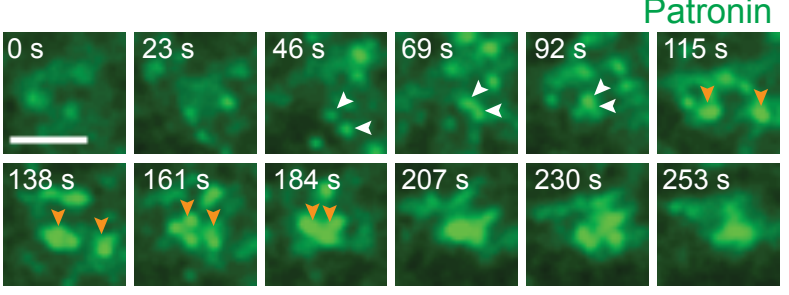

C

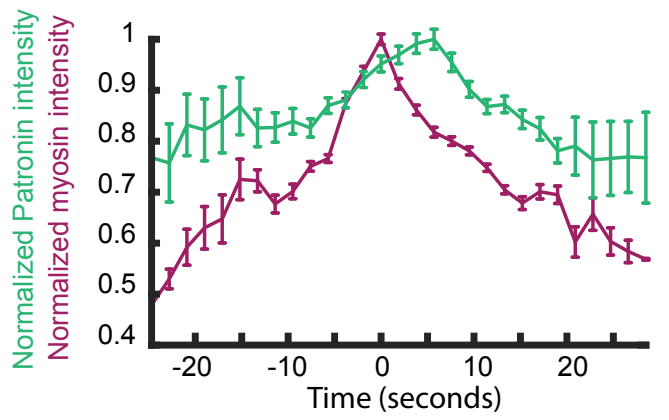

E

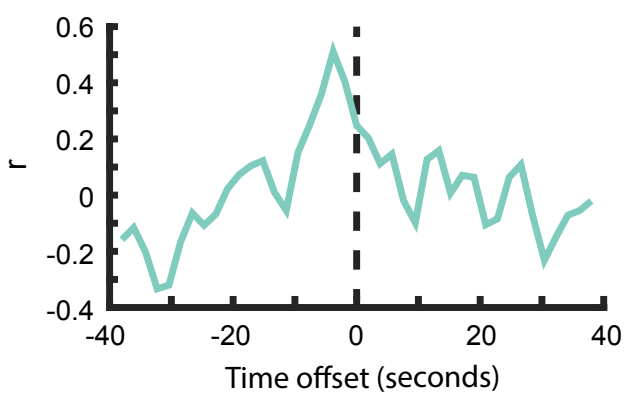

B

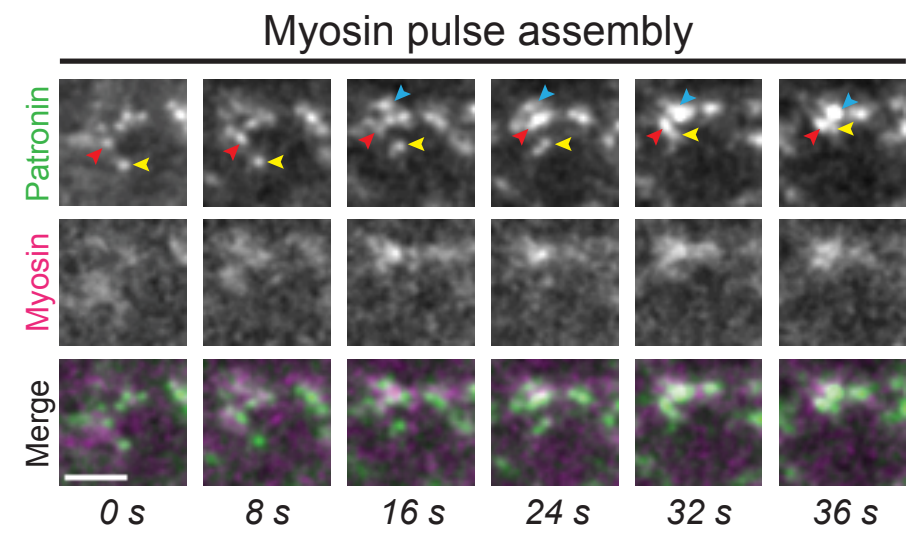

D

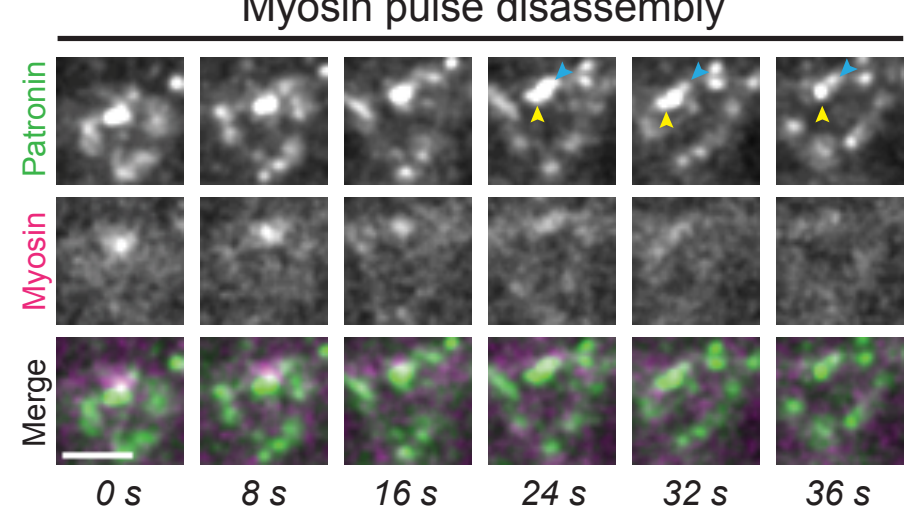


pioRxiv preprint doi: https://doi.org/10.1101/540344; this version posted February 4, 2019. The copyright holder for this preprint (which was not

Figure 4certified by peer review) is the author/funder, who has granted bioRxiv a license to display the preprint in perpetuity. It is made available under A aCC-BY-NC-ND 4.0 Inte $B^{\text {ational license. }}$
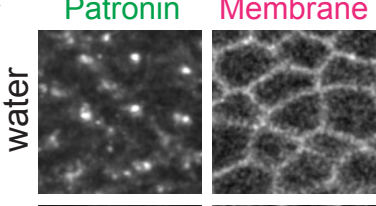

Merge
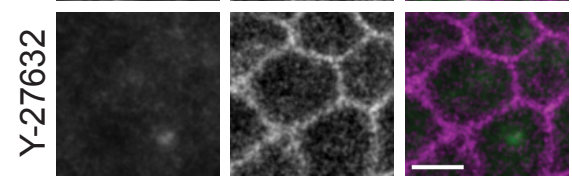

C Patronin Membrane

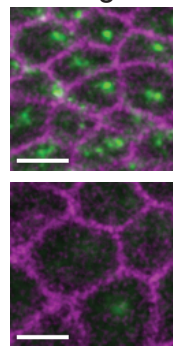

\section{Merge}

$\stackrel{\infty}{\mathscr{m}}$
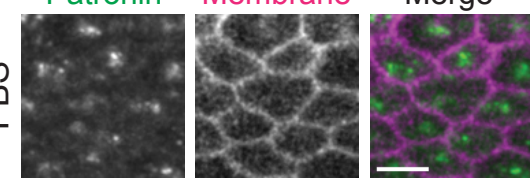

ֻु
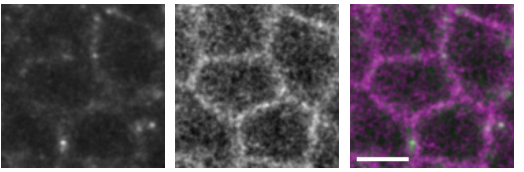

$\mathbf{E}$

\section{DMSO}
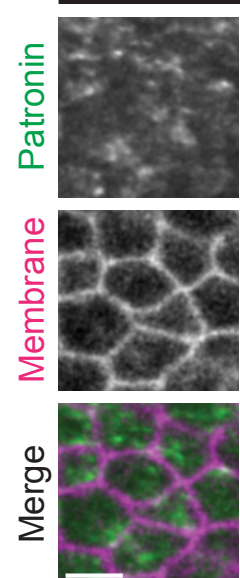

0 min
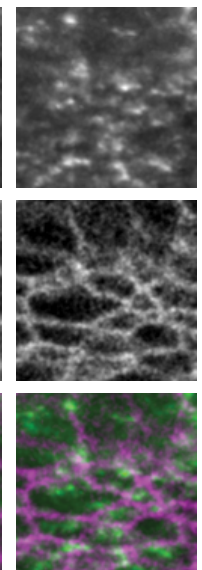

$2.5 \mathrm{~min}$
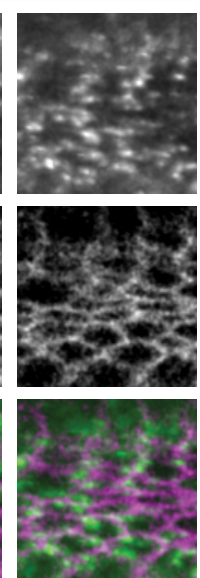

5 min

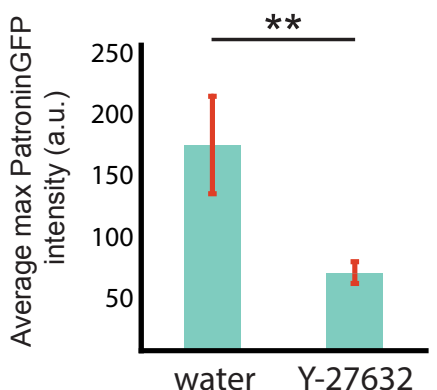

D

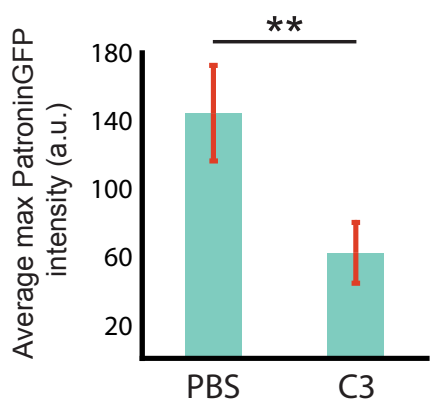

CytoD
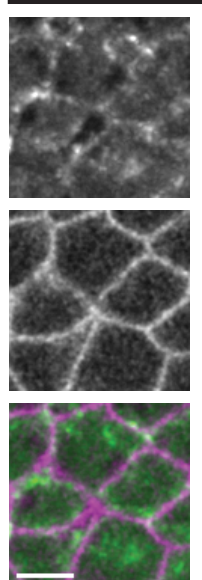

0 min
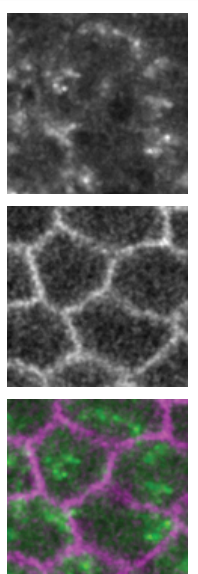

3 min
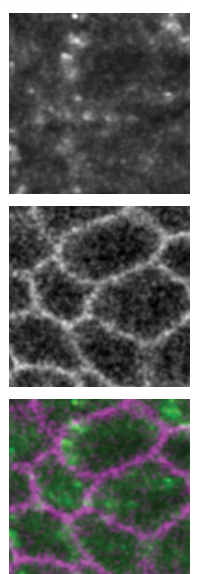

6 min 


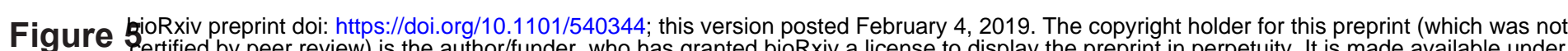
ertified by peer review) is the author/funder, who has granted bioRxiv a license to display the preprint in perpetuity. It is made available under

A
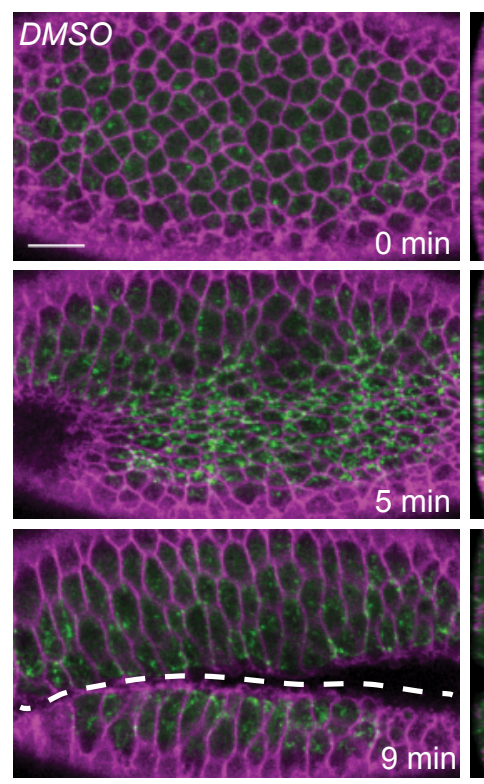

B

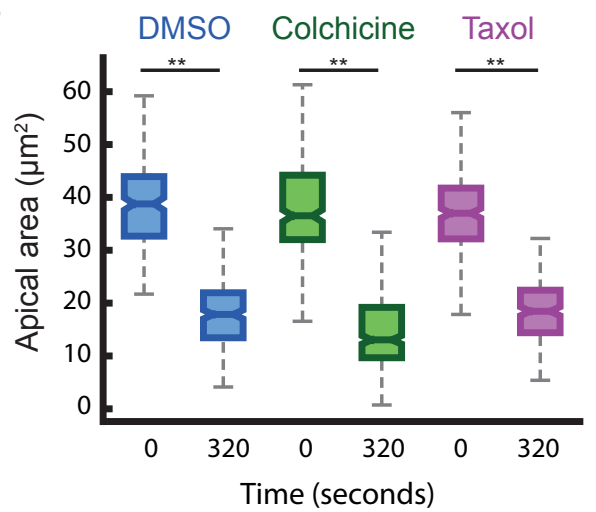

D

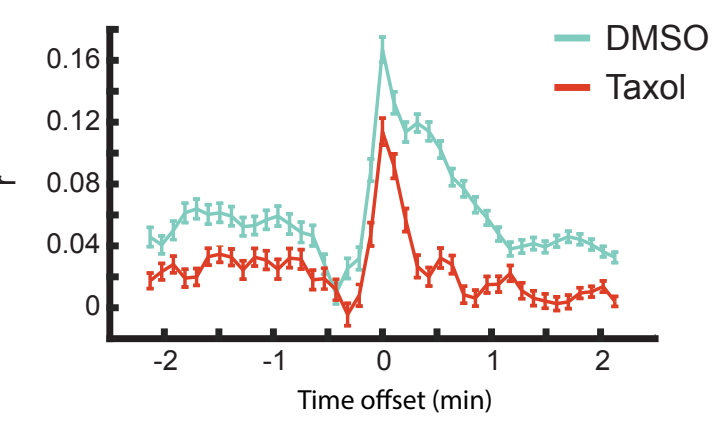

E

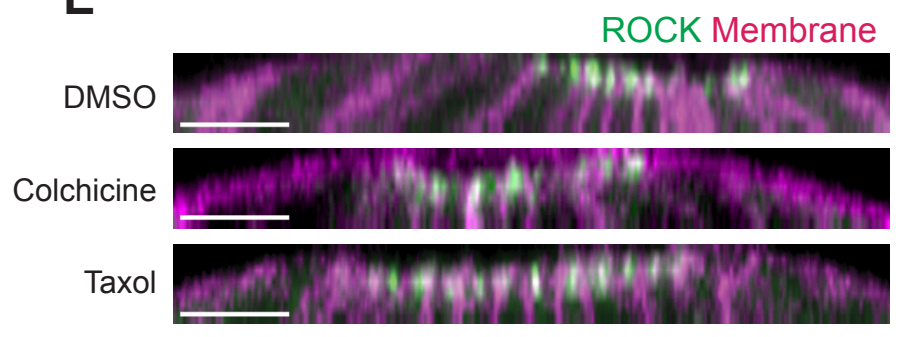

C
Myosin Membrane
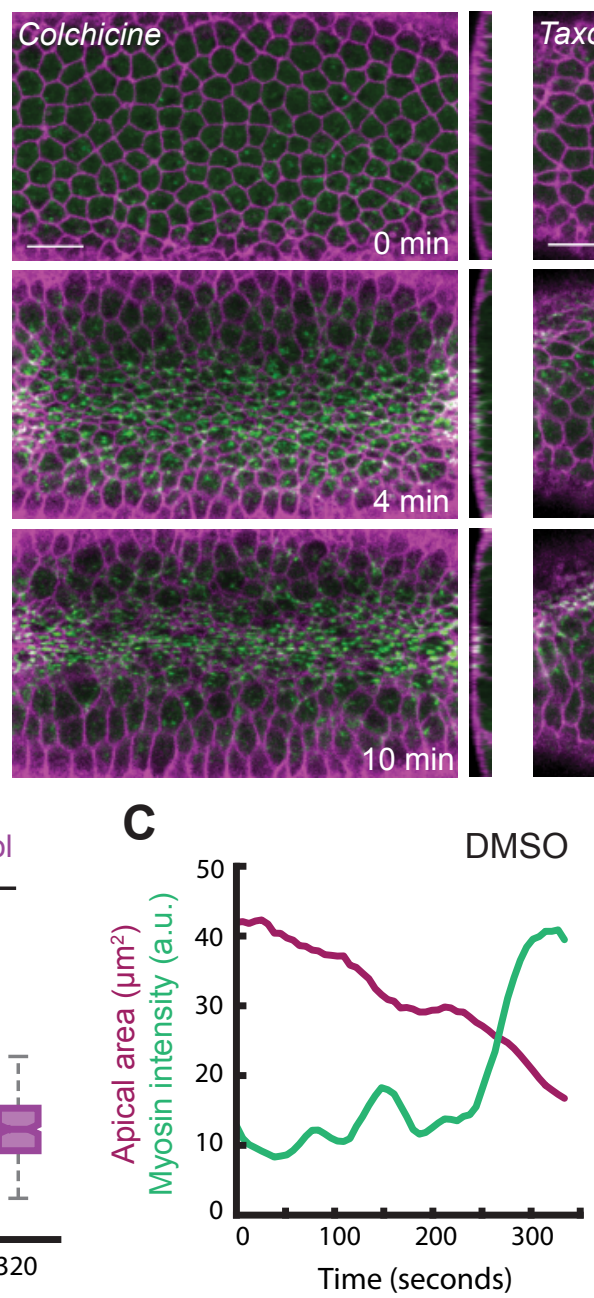

$\mathbf{F}$
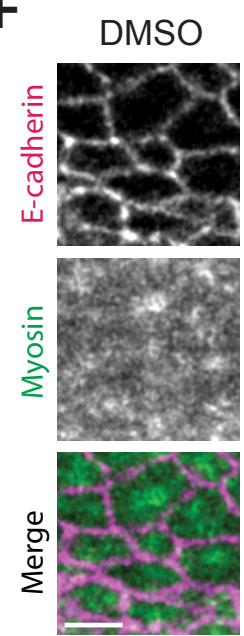

$\mathrm{yz}$

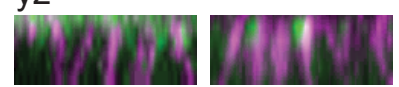

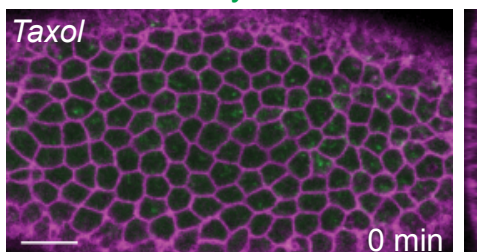
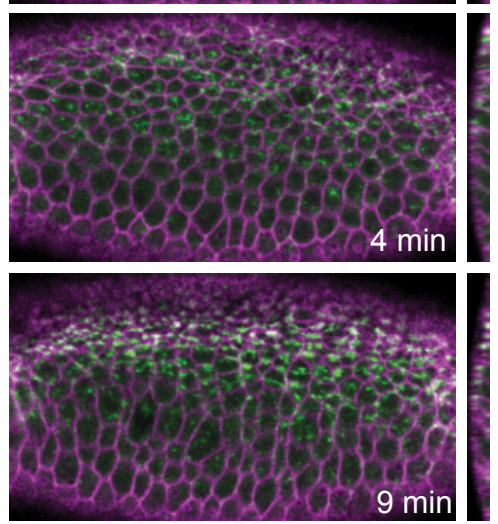

$9 \mathrm{~min}$

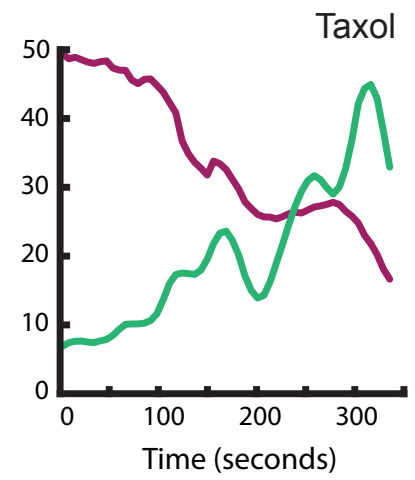

Colchicine Taxol
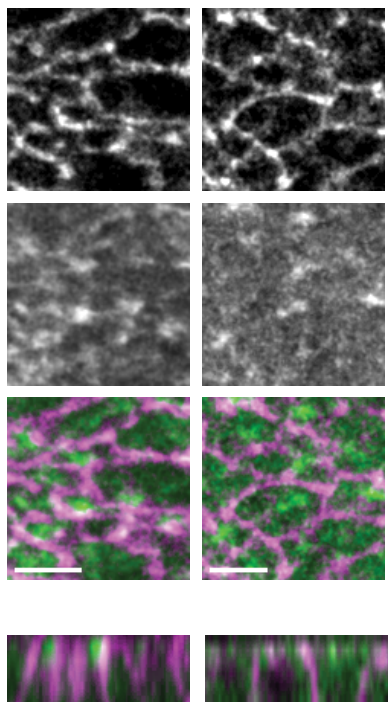
A
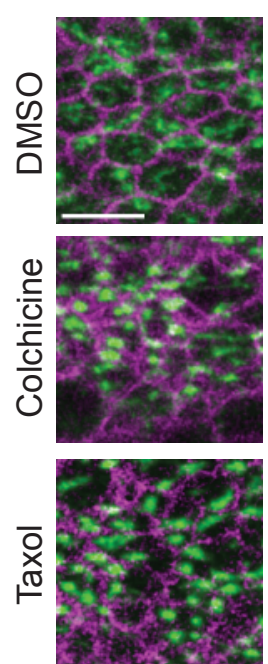

$0 \mathrm{~s}$

C

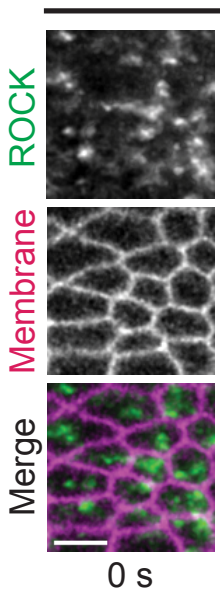

D

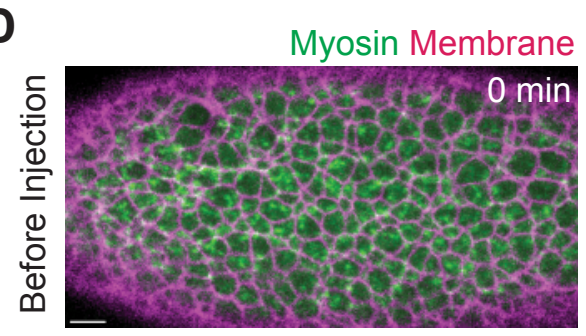

+Colchicine @ T=20 s
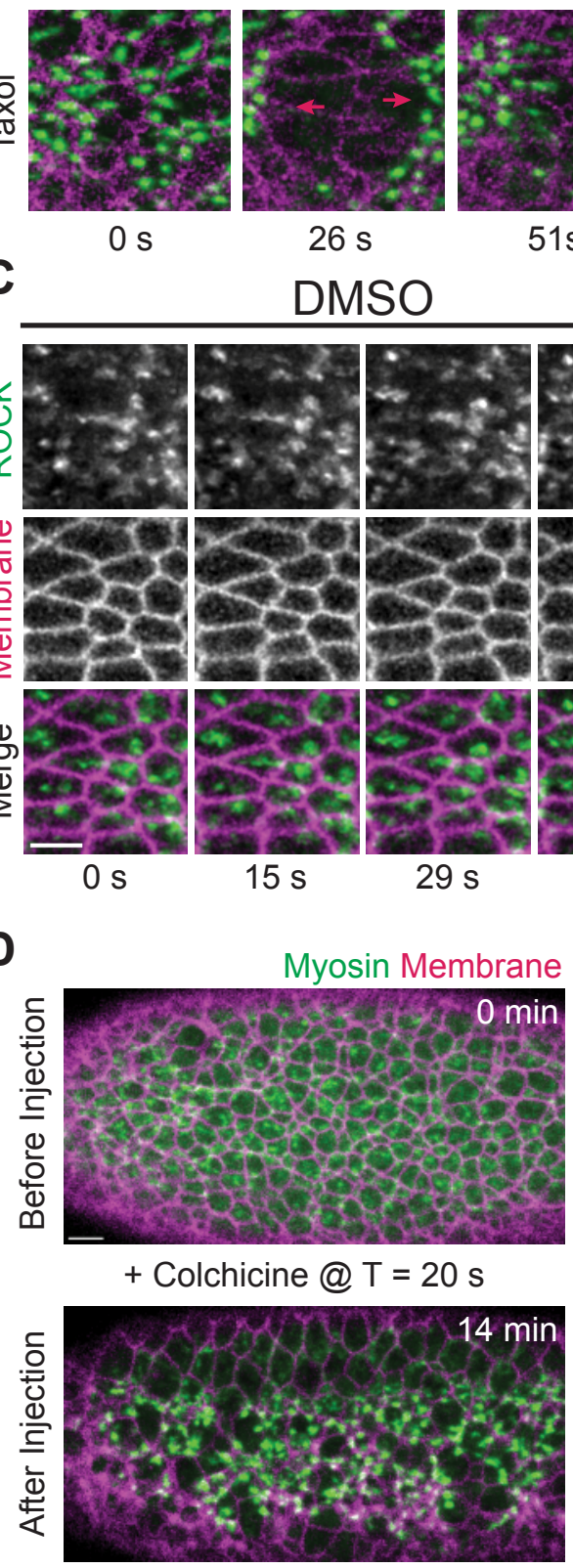

$26 \mathrm{~s}$

DMSO

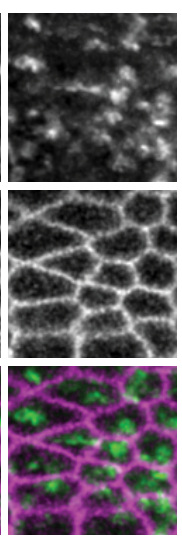

$15 \mathrm{~s}$
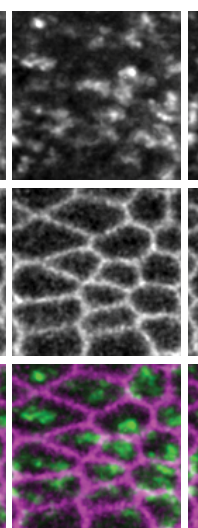

$29 \mathrm{~s}$
B
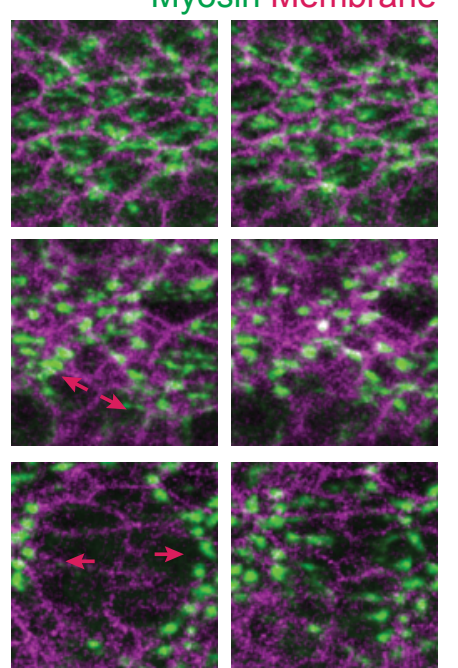

$51 \mathrm{~s}$

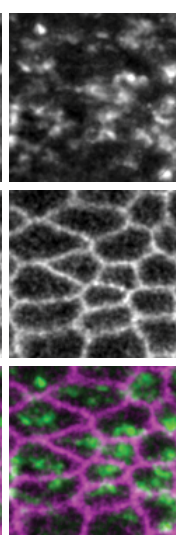

$44 \mathrm{~s}$

E
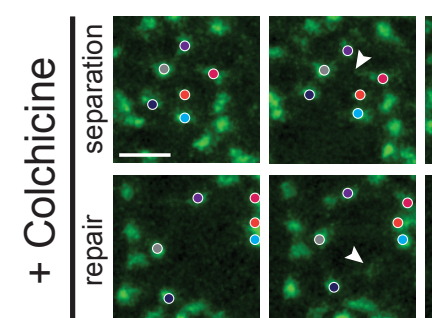

Myosin

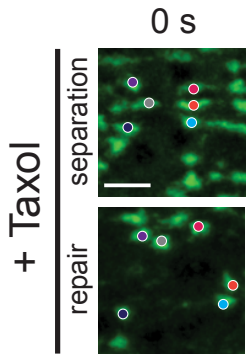

$12 \mathrm{~s}$
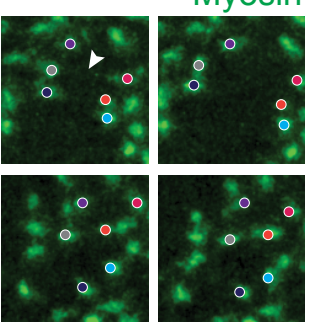

$24 \mathrm{~s}$

$36 \mathrm{~s}$
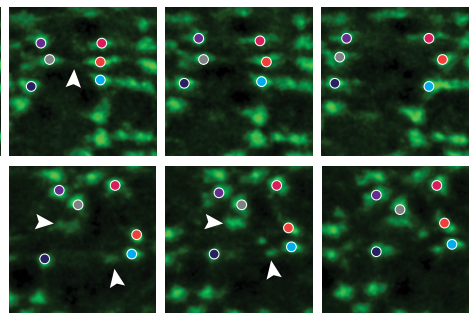

Taxol

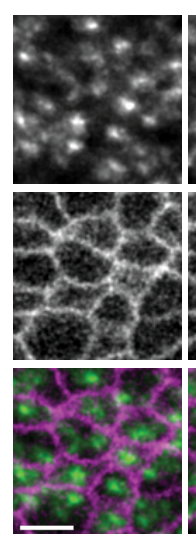

$0 \mathrm{~s}$

$15 \mathrm{~s}$

$29 \mathrm{~s}$

$44 \mathrm{~s}$

Myosin
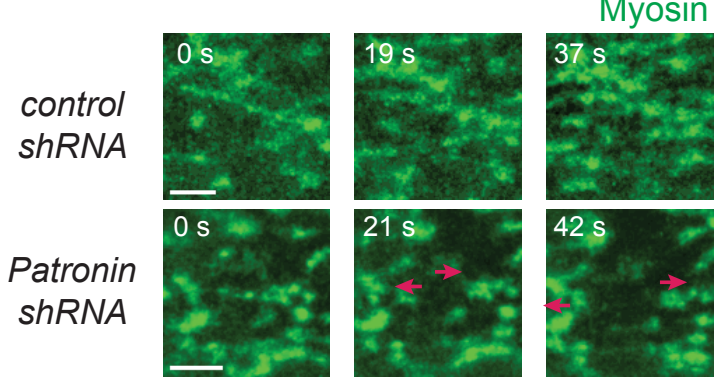

$\mathbf{F}$

- Adherens junction $\succ$ Actin + Myosin

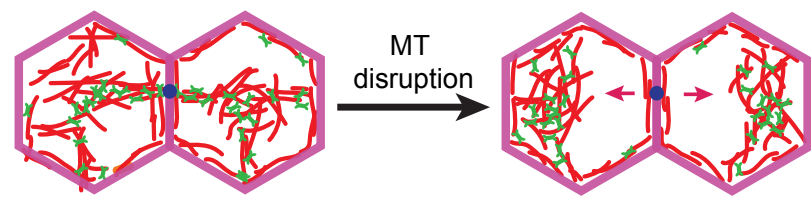


A
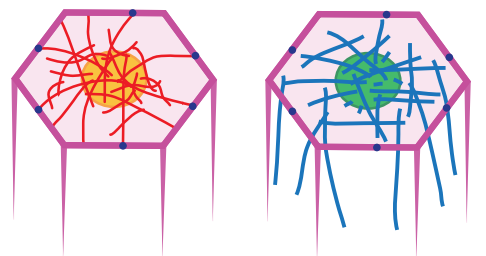

- E-cadherin

C3
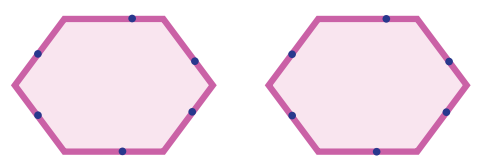

$\sim$ F-actin

Microtubule

CytoD
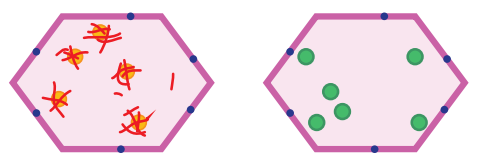

RhoA / ROCK

LatB 更
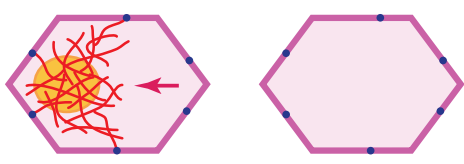

Patronin

Colchicine

B

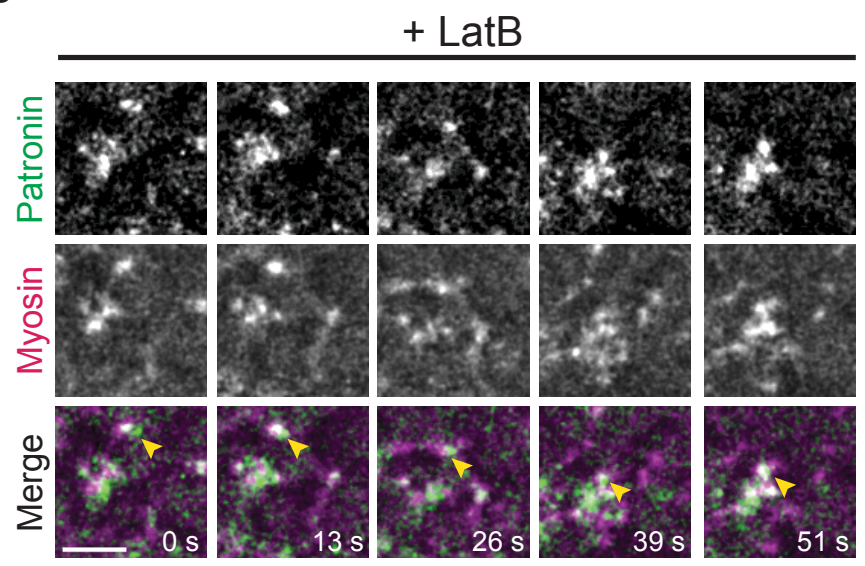


A
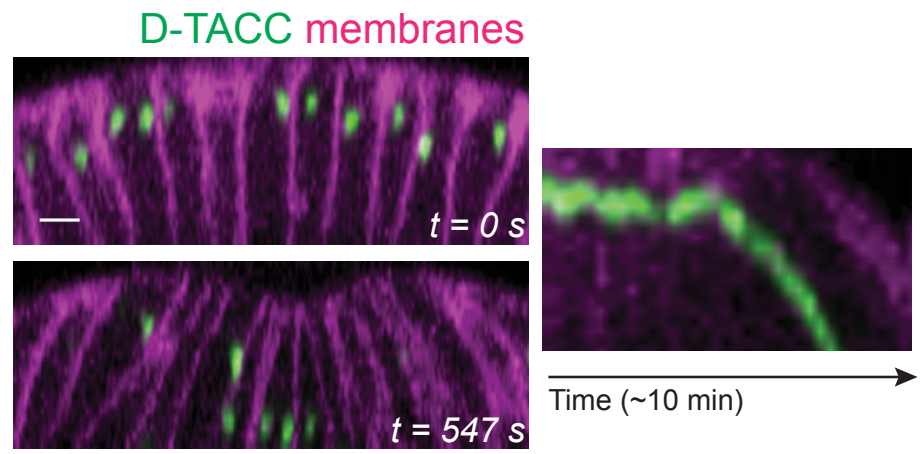

B

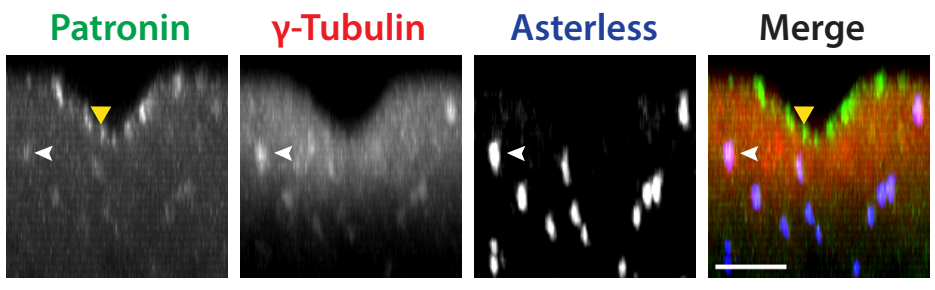

C

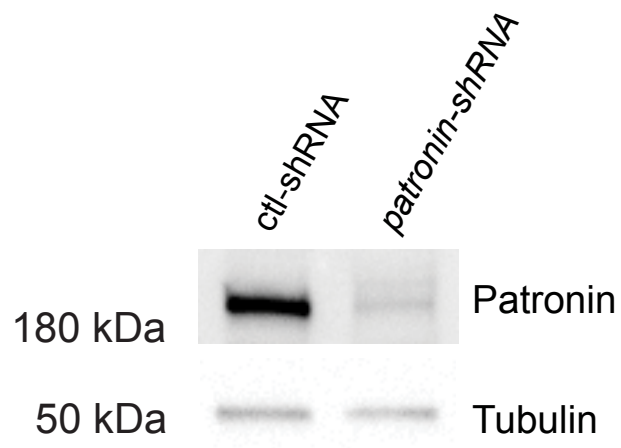


bioRxiv preprint doi: https://doi.org/10.1101/540344; this version posted February 4, 2019. The copyright holder for this preprint (which was not

Figure Eezified by peer review) is the author/funder, who has granted bioRxiv a license to display the preprint in perpetuity. It is made available under aCC-BY-NC-ND 4.0 International license.

A

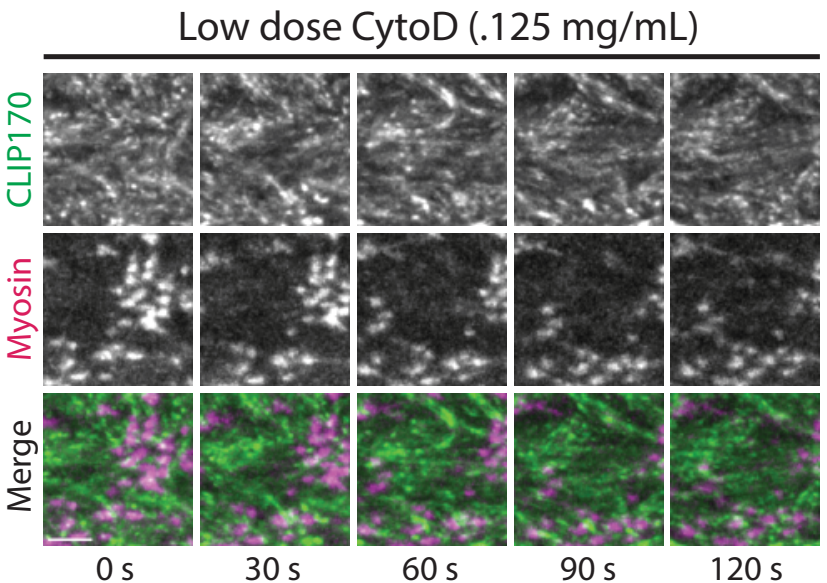

High dose CytoD $(5 \mathrm{mg} / \mathrm{mL})$

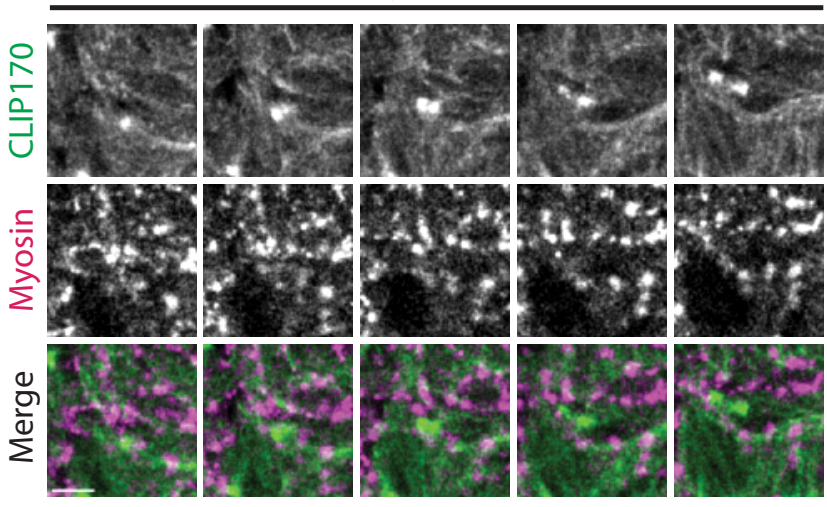

$0 \mathrm{~s}$

$60 \mathrm{~s}$

$90 \mathrm{~s}$

$120 s$

B

Patronin Membrane
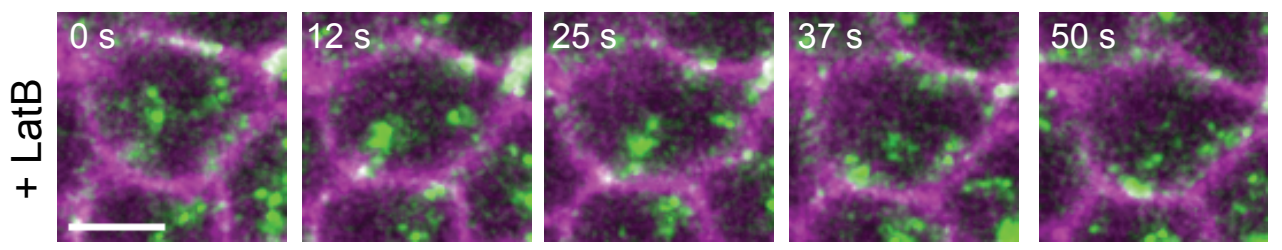
bioRxiv preprint doi: https://doi.org/10.1101/540344; this version posted February 4, 2019. The copyright holder for this preprint (which was not Figurected by peer review) is the author/funder, who has granted bioRxiv a license to display the preprint in perpetuity. It is made available under aCC-BY-NC-ND 4.0 International license.

A
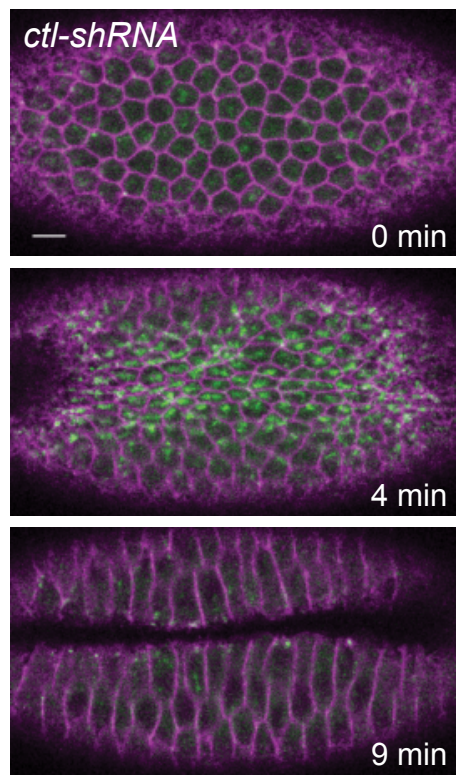

Membrane Myosin
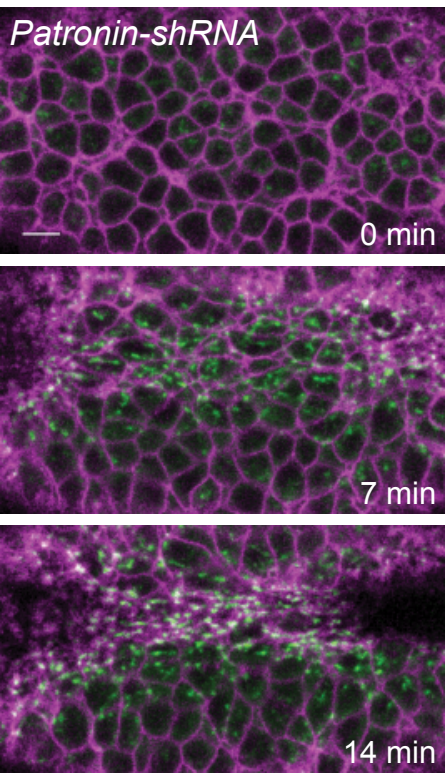

B ctrl shRNA

Patronin shRNA

mid-cellularization
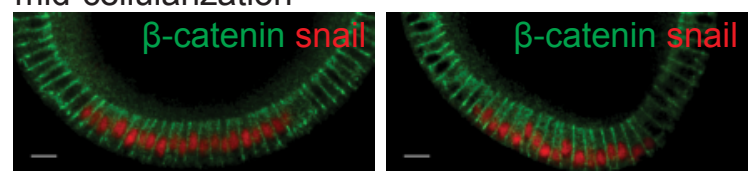

late cellularization
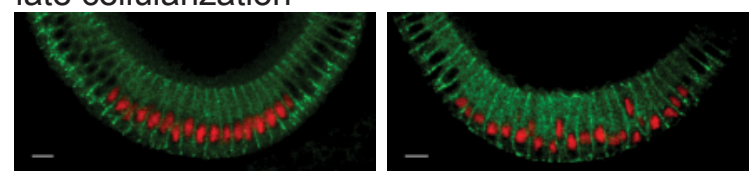

gastrulation
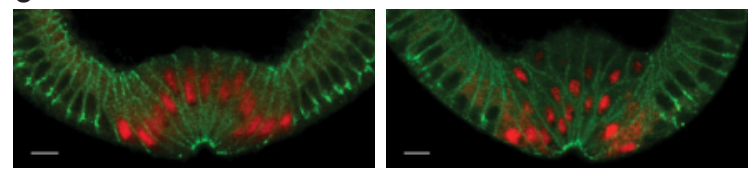

C control shRNA
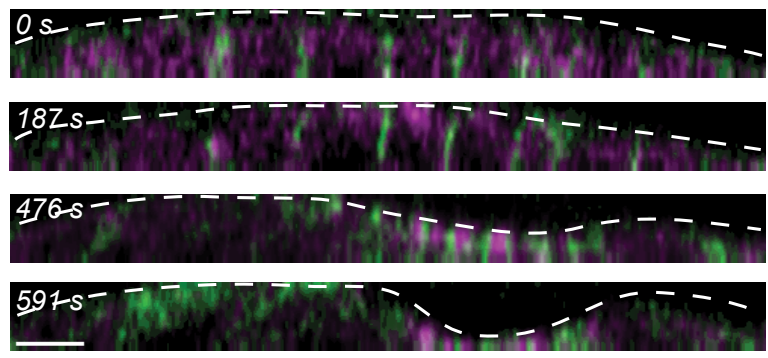

Patronin shRNA

E-cadherin Myosin
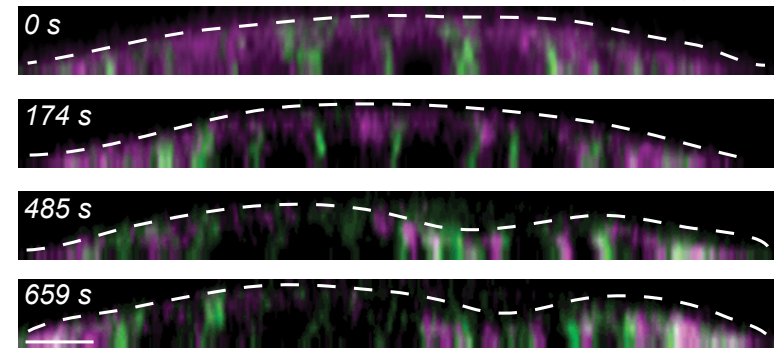

D
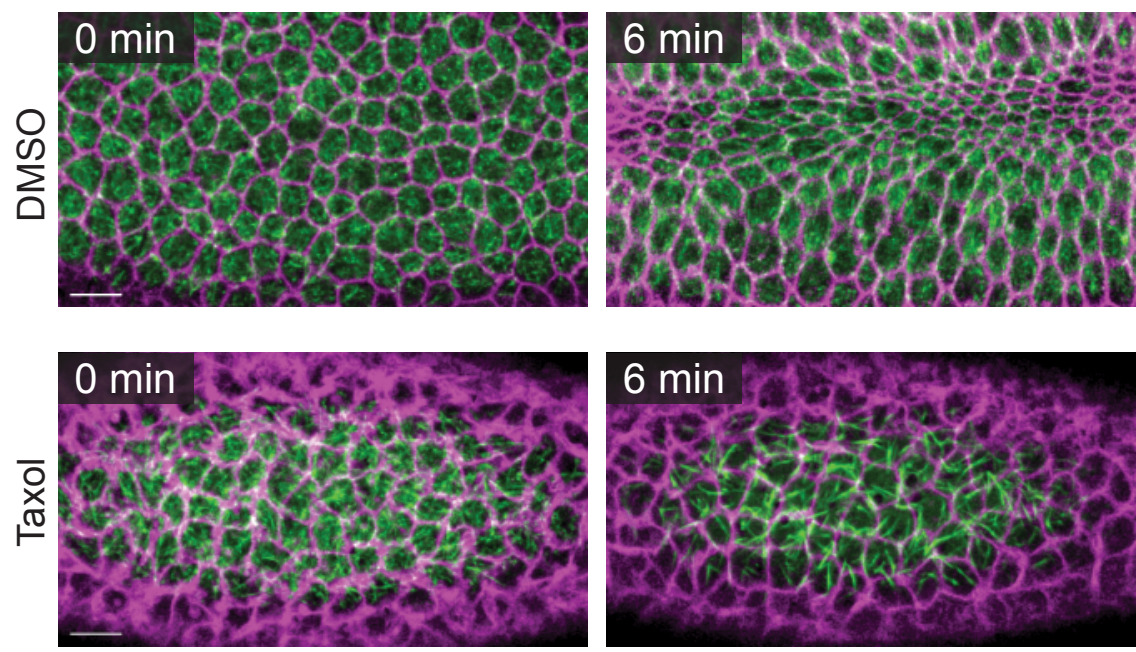

CLIP170 Membrane
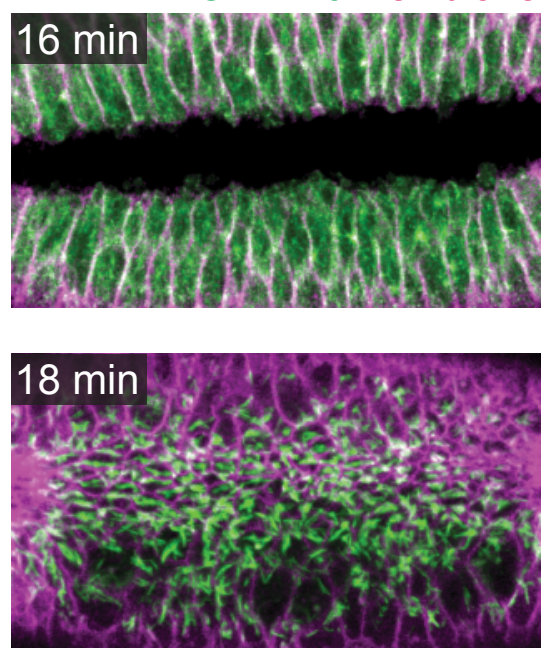
bioRxiv preprint doi: https://doi.org/10.1101/540344; this version posted February 4, 2019. The copyright holder for this preprint (which was not Figurec8t4ied by peer review) is the author/funder, who has granted bioRxiv a license to display the preprint in perpetuity. It is made available under
aCC-BY-NC-ND 4.0 International license. A

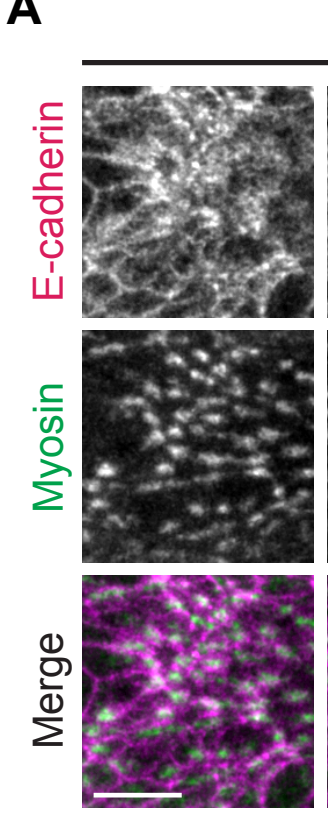

$0 \mathrm{~s}$
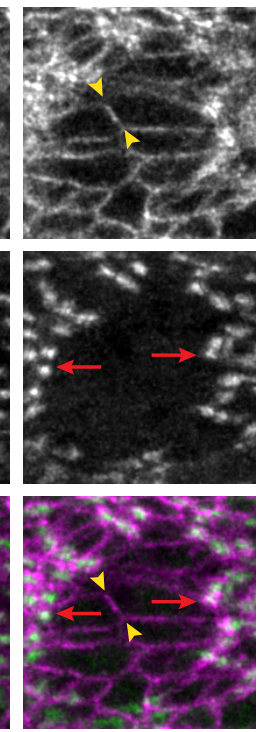

$12 \mathrm{~s}$
+ Taxol

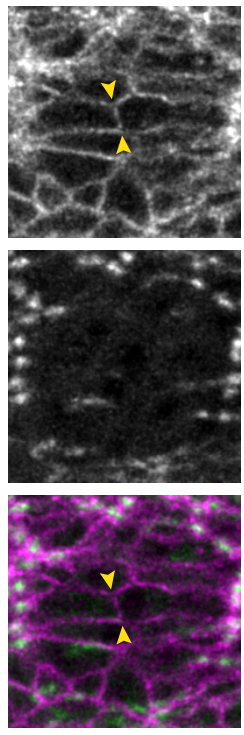

$25 \mathrm{~s}$
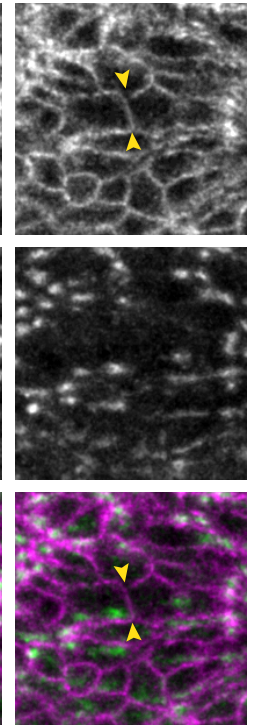

$37 \mathrm{~s}$
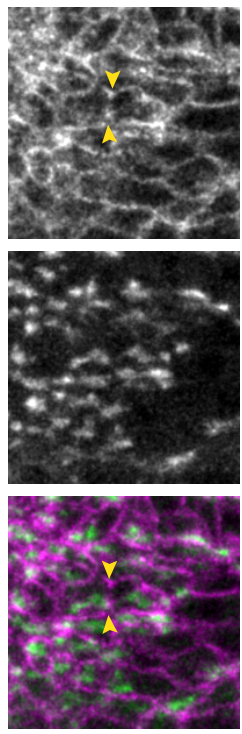

$50 \mathrm{~s}$

B

E-cadherin localization
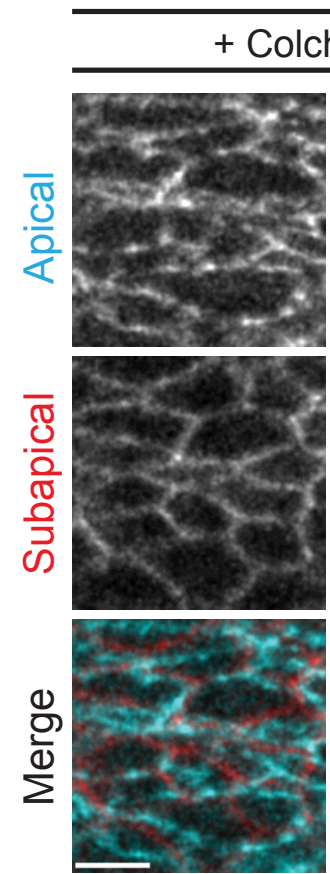

$0 \mathrm{~min}$
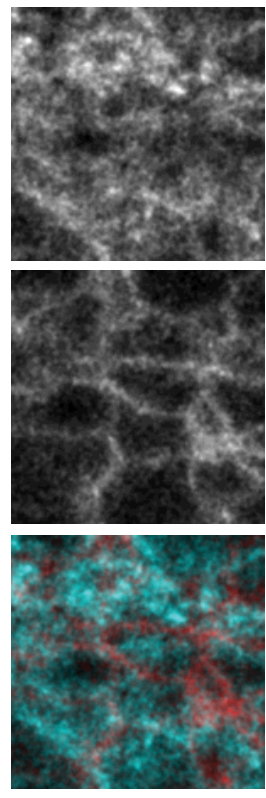

$10 \min$
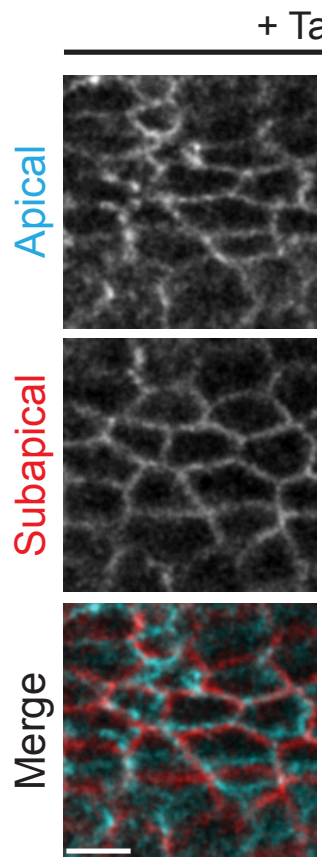

$0 \mathrm{~min}$
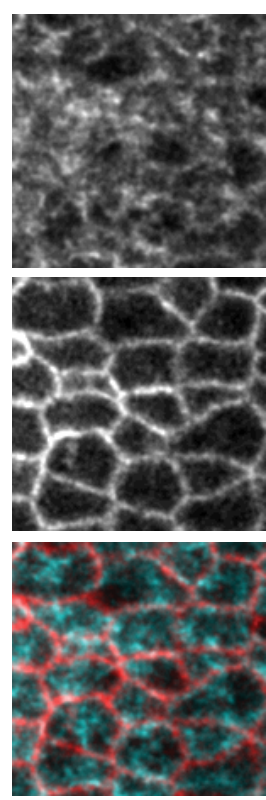

$7 \mathrm{~min}$ 
bioRxiv preprint doi: https://doi.org/10.1101/540344; this version posted February 4, 2019. The copyright holder for this preprint (which was not certified by peer review) is the author/funder, who has granted bioRxiv a license to display the preprint in perpetuity. It is made available under aCC-BY-NC-ND 4.0 International license.

Table S1

Fly Stocks Used

\begin{tabular}{|c|c|c|}
\hline Stock & Genotype & Source \\
\hline 1 & $\mathrm{P}\{\mathrm{w}[+\mathrm{mC}]=$ Ubi-p63E-Patronin.A.GFP $\} 7 \mathrm{~F}, \mathrm{w}\left[{ }^{*}\right]$ & Bloomington Drosophila Stock Center \\
\hline 2 & OreR & Bloomington Drosophila Stock Center \\
\hline 3 & w; gap43::mCherry-7/TM3 & Bardet et al., 2013 \\
\hline 4 & w; mat67; Gap43::mCherry/TM3, Ser [1] & \\
\hline 5 & w; mat67, Sqh::Cherry[B1]; Dr/TM3, Sb[1] & \\
\hline 6 & w; mat67; Sb/TM3, Ser [1] & \\
\hline 7 & w; mat67, Sqh::Cherry[B1]; GFP::CLIP170/TM3, Sb [1] & \\
\hline 8 & w; mat67, Sqh::GFP; mat15, Gap43::mCherry/TM3, Sb[1] & Vasquez et al., 2014 \\
\hline 9 & y, w; mat67, Sqh::mCherry; mat15, E-cadherin::GFP & Mason et al., 2016 \\
\hline 10 & $y[1] \mathrm{sc}\left[^{*}\right] \mathrm{v}[1] ; \mathrm{P}\{\mathrm{y}[+\mathrm{t} 7.7] \mathrm{v}[+\mathrm{t} 1.8]=$ TRiP.HMS01547\}attP2 & Bloomington Drosophila Stock Center \\
\hline 11 & $\mathrm{y}[1] \mathrm{sc}\left[{ }^{\star}\right] \mathrm{v}[1] ; \mathrm{P}\{\mathrm{y}[+\mathrm{t} 7.7] \mathrm{v}[+\mathrm{t} 1.8]=$ TRiP.GL01052 $\}$ attP2 & Bloomington Drosophila Stock Center \\
\hline 12 & $\mathrm{P}\left\{\mathrm{w}\left[+\mathrm{m}^{*}\right]=\right.$ Ubi-tacc.GFP $\} 1, \mathrm{y}[1] \mathrm{w}\left[{ }^{*}\right]$ & Bloomington Drosophila Stock Center \\
\hline 13 & Ubi>GFP::rok, gap43::mCherry (X) & \\
\hline 14 & $y, w ;$ sqhAX3;P\{w+ sqh::GFP $\} 42$ & Bloomington Drosophila Stock Center \\
\hline 15 & w; Gap43::mCherry(attp40); Sqh::GFP & Martin et al., 2010 \\
\hline 16 & P\{Ubi-Tacc.GFP $\} 1, y, w$ & Bloomington Drosophila Stock Center \\
\hline 17 & y, w; UAS-GFP::CLIP170/TM3, Sb & \\
\hline
\end{tabular}

Crosses (F2 embryos used)

Stocks Used (Female $x$ Male) Figure

\begin{tabular}{|c|l|}
\hline $1 \times 3$ & $1, \mathrm{~B}$ and $\mathrm{C} ; 2 \mathrm{E} ; 4, \mathrm{~A}-\mathrm{E} ; \mathrm{S} 2 \mathrm{~B}$ \\
\hline $1 \times 5$ & $1 \mathrm{~F} ; 3, \mathrm{~B}-\mathrm{D} ; 7 \mathrm{~B}$ \\
\hline $1 \times 6$ & $1 \mathrm{G}$ \\
\hline 1 sib cross & $3 \mathrm{~A} ; \mathrm{S} 1 \mathrm{~B}$ \\
\hline $11 \times 7$ & $2 \mathrm{C} ; \mathrm{S} 2 \mathrm{~A}$ \\
\hline $14 \times 15$ & $5 \mathrm{~A}-\mathrm{D} ; 6, \mathrm{~A}, \mathrm{~B}$, and D \\
\hline $11 \times 9$ & $5 \mathrm{~F} ; \mathrm{S} 3 \mathrm{C} ; \mathrm{S} 4, \mathrm{~A}$ and B \\
\hline $11 \times 8$ & $6 \mathrm{E} ; \mathrm{S} 3 \mathrm{~A}$ \\
\hline $10 \times 8$ & $6 \mathrm{E} ; \mathrm{S} 3 \mathrm{~A}$ \\
\hline $11 \times 6$ & $2 \mathrm{D} ; \mathrm{S} 1 \mathrm{C} ; \mathrm{S} 3 \mathrm{~B}$ \\
\hline $10 \times 6$ & $2 \mathrm{D} ; \mathrm{S} 1 \mathrm{C} ; \mathrm{S} 3 \mathrm{~B}$ \\
\hline 13 sib cross & $5 \mathrm{E} ; 6 \mathrm{C}$ \\
\hline $16 \times 3$ & S1 A \\
\hline $17 \times 3$ & S3 D \\
\hline 2 sib cross & $2, \mathrm{~A}$ and B \\
\hline
\end{tabular}

